\title{
Families of monotone symplectic manifolds constructed via symplectic cut and their Lagrangian submanifolds
}

\author{
Agnes Gadbled
}

\begin{abstract}
We describe families of monotone symplectic manifolds constructed via the symplectic cutting procedure of Lerman [14] from the cotangent bundle of manifolds endowed with a free circle action. We also give obstructions to the monotone Lagrangian embedding of some compact manifolds in these symplectic manifolds.
\end{abstract}

53D05, 53D12; 53D20, 53D40

\section{Introduction}

If $V$ is a closed manifold endowed with a free circle action, this action induces a Hamiltonian action of the circle on the cotangent bundle $T^{*} V$ of $V$. If $H$ denotes the associated Hamiltonian vanishing on the zero section of $T^{*} V, W_{\xi}$ the symplectic manifold obtained by symplectic cut of $T^{*} V$ at the level $\xi$ and $Q_{\xi}$ the reduced manifold $H^{-1}(\xi) / \mathbb{S}^{1}$, we prove that $W_{\xi}$ has the following properties:

(1) (Lemma 2.7) The manifold $W_{\xi}$ is a complex line bundle over $Q_{\xi}$.

(2) (Proposition 2.5) The quotient $Q_{\xi}$ is a symplectic submanifold of $W_{\xi}$ diffeomorphic to the cotangent bundle of $B=V / \mathbb{S}^{1}$. If $e$ is the Euler class of the circle bundle $V \rightarrow B$ and $q$ is the projection $Q_{\xi} \rightarrow B$, then the class of the symplectic form over $Q_{\xi}$ is $-2 \pi \xi q^{*} e$.

(3) (Proposition 2.6) The class in $H^{2}\left(W_{\xi} ; \mathbb{R}\right)$ of the symplectic form of $W_{\xi}$ is related to the first Chern class of $W_{\xi}$ by the formula

$$
\left[\omega_{\xi}\right]=-2 \pi \xi c_{1}\left(W_{\xi}\right) .
$$

In particular, $W_{\xi}$ is monotone for negative $\xi$.

(4) (Proposition 4.5) The manifold $W_{\xi}$ is endowed with a Hamiltonian circle action and the sum of the weights of this action at a fixed point is equal to 1 .

(5) (Theorem 3.3) The zero section $V$ of the cotangent bundle can be embedded as a monotone Lagrangian submanifold of $W_{\xi}$ for any negative level $\xi$. 
(6) (Corollary 4.10) Any exact compact Lagrangian submanifold of $T^{*} V$ with vanishing Maslov class can be embedded as a monotone Lagrangian submanifold of $W_{\xi}$ for some negative level $\xi$.

In the general case of a monotone Lagrangian submanifold in a symplectic manifold, Seidel [21] has proved, under some assumptions on the symplectic manifold and the Lagrangian submanifold, that the Floer homology can be absolutely graded. He also proved that if the symplectic manifold is endowed with a Hamiltonian circle action, the Floer homology of the Lagrangian submanifold is periodic with respect to the absolute grading with period the sum of the weights of the action at a fixed point. Applying these results to the monotone Lagrangian submanifolds of the symplectic cut $W_{\xi}$, we get:

(1) (Theorem 4.8) An obstruction result on the embedding of a compact simply connected manifold as a monotone Lagrangian submanifold in a symplectic cut under conditions on the Euler class $e$.

(2) (Theorems 4.11 and 4.12) Results on the index of the fundamental group of an exact compact Lagrangian submanifold with vanishing Maslov class in the cotangent bundle of a manifold endowed with a free circle action.

(3) (Theorem 4.20) The monotone Lagrangian tori in a simply connected symplectic cut have a Maslov number equal to 2 .

In Section 1, we explain the symplectic cutting construction for our specific circle action and describe it in some examples. In the Section 2, we study the structure of the spaces which appear in the construction and their monotonicity. Section 3 is essentially devoted to the monotonicity of the canonical embedding of $V$ in the symplectic cut. After briefly recalling Seidel's periodicity results, we apply them to the monotone Lagrangian submanifolds of the symplectic cut in Section 4 and prove the results stated above. Appendices A and B contain complements to some proofs and a discussion of the monotonicity in the case of a semifree circle action.

Acknowledgements This is a part of the author's PhD thesis at the University of Strasbourg. I would like to thank Michèle Audin and Mihai Damian for their helpful suggestions and comments and for their careful reading of a earlier version of this article. I am greatly indebted to them for the time they spend discussing this subject with me.

The author was partially supported by ANR project "Floer Power" ANR-08-BLAN0291-03, SNF project "Complexity and recurrence in Hamiltonian systems" and MSRI postdoc fellowship NSF grant DMS-0441170. 


\section{Symplectic cut of cotangent bundles}

Let $\pi: V \rightarrow B$ be a principal circle bundle, the base $B$ being path connected. Denote by $X$ the fundamental vector field associated with the circle action on $V$. As this $\mathbb{S}^{1}$-action is free, the fundamental vector field cannot vanish.

Remark 1.1 We refer to Appendix B for the study of the case of a semifree circle action.

Denote by $e \in H^{2}(B ; \mathbb{Z})$ the Euler class of the principal bundle $\pi$ and by $N_{e}$ the nonnegative generator of the subgroup $\left\langle e, \pi_{2}(B)\right\rangle$ of $\mathbb{Z}$.

Example 0 In the case of the trivial bundle $V=B \times \mathbb{S}^{1} \rightarrow B, \pi$ is the projection on the first factor, the circle action is the complex multiplication on the factor $\mathbb{S}^{1}$ (seen as the group of complex numbers of module 1) and the Euler class is zero.

A subcase is the case of $\mathbb{S}^{1}$ acting on itself by multiplication, the quotient $B$ being reduced to a point.

Example 1 If $V=\mathbb{S}^{2 n+1} \rightarrow B=\mathbb{C P}^{n}(n \geq 1)$ is the Hopf bundle, the Euler class is equal to minus one times the preferred generator of $H^{2}\left(\mathbb{C P} \mathbb{P}^{n} ; \mathbb{Z}\right.$ ) (this can also be considered as a choice for the preferred generator of $H^{2}(\mathbb{C P} ; \mathbb{Z})$ ).

Example 2 Let $V$ be the lens space $L_{p}^{2 n+1}$, quotient of the sphere $\mathbb{S}^{2 n+1}$ by the action of the subgroup $\mathbb{Z} / p$ of $\mathbb{S}^{1}$ consisting of the $p$-roots of unity.

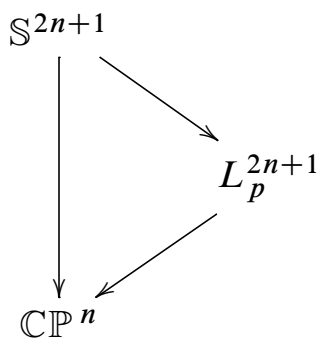

The free action of $\mathbb{S}^{1}$ on $\mathbb{S}^{2 n+1}$ enables us to define a free action of $\mathbb{S}^{1}$ on $L_{p}^{2 n+1}$, with quotient $\mathbb{C P}^{n}$, such that the Euler class of the principal circle bundle $L_{p}^{2 n+1} \rightarrow \mathbb{C P}^{n}$ is $-p$ times the preferred generator of $H^{2}\left(\mathbb{C P}^{n} ; \mathbb{Z}\right)$ (chosen in Example 1).

Example 3 We can also consider the Stiefel manifold $V_{2}\left(\mathbb{R}^{n+2}\right)$ consisting of the pairs of orthonormal vectors of $\mathbb{R}^{n+2}$. This manifold $V_{2}\left(\mathbb{R}^{n+2}\right)$ can also be described as the quotient $\mathrm{SO}(n+2) / \mathrm{SO}(n)$. The subgroup $\mathrm{SO}(2) \simeq \mathbb{S}^{1}$ of $\mathrm{SO}(n+2)$ acts freely 
on $V_{2}\left(\mathbb{R}^{n+2}\right)$, the quotient of this action being the Grassmannian $B=\widetilde{G}_{2}\left(\mathbb{R}^{n+2}\right)$ of the (2-dimensional) oriented planes in $\mathbb{R}^{n+2}$.

This Grassmannian can be identified with the quadric $Q^{n}$ in $\mathbb{C P}^{n+1}$ of equation

$$
\sum_{j=0}^{n+1} z_{i}^{2}=0 .
$$

To see this, we can use the first description of the Stiefel manifold $V_{2}\left(\mathbb{R}^{n+2}\right)$ as a subset of $\left(\mathbb{R}^{n+2}\right)^{2}$ :

$$
V_{2}\left(\mathbb{R}^{n+2}\right)=\left\{(x, y) \in\left(\mathbb{R}^{n+2}\right)^{2} \mid x \cdot y=0,\|x\|=\|y\|=1\right\} .
$$

The action of $\mathrm{SO}(2) \simeq \mathbb{S}^{1}$ can then be written as

$$
e^{i \theta} \cdot(x, y)=(\cos (\theta) x-\sin (\theta) y, \sin (\theta) x+\cos (\theta) y) .
$$

The map

$$
\begin{aligned}
V_{2}\left(\mathbb{R}^{n+2}\right) & \longrightarrow \mathbb{C P}^{n+1} \\
(x, y) & \longmapsto[x+i y]
\end{aligned}
$$

descends to the quotient and defines an embedding of the Grassmannian $\widetilde{G}_{2}\left(\mathbb{R}^{n+2}\right)$ in $\mathbb{C} \mathbb{P}^{n+1}$, the image of which is the quadric $Q^{n}$ (see Audin [3]).

For $n \geq 3$, by the Lefschetz hyperplane theorem, the embedding of the quadric induces an isomorphism between $H^{2}\left(\widetilde{G}_{2}\left(\mathbb{R}^{n+2}\right) ; \mathbb{Z}\right)$ and $H^{2}\left(\mathbb{C} \mathbb{P}^{n+1} ; \mathbb{Z}\right)$. Therefore, $H^{2}\left(\widetilde{G}_{2}\left(\mathbb{R}^{n+2}\right) ; \mathbb{Z}\right)$ is isomorphic to $\mathbb{Z}$, a preferred generator being given by the pullback of the preferred generator of $H^{2}\left(\mathbb{C P}^{n+1} ; \mathbb{Z}\right)$. Besides, $V_{2}\left(\mathbb{R}^{n+2}\right)$ is the total space of the pullback of the Hopf bundle $\mathbb{S}^{2 n+3} \rightarrow \mathbb{C P}^{n+1}$ by the embedding $j$ of $\widetilde{G}_{2}\left(\mathbb{R}^{n+2}\right)$ in $\mathbb{C P} \mathbb{P}^{n+1}$ :

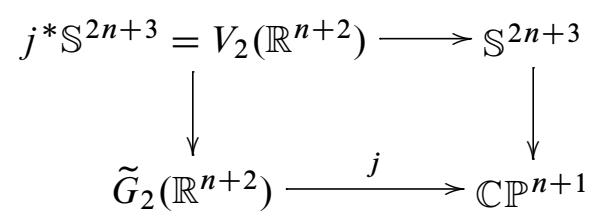

Indeed, with the map $V_{2}\left(\mathbb{R}^{n+2}\right) \rightarrow \mathbb{S}^{2 n+3}$ given by $(x, y) \mapsto(1 / \sqrt{2})(x+i y)$ and the projection $V_{2}\left(\mathbb{R}^{n+2}\right) \rightarrow \widetilde{G}_{2}\left(\mathbb{R}^{n+2}\right)$, the above diagram commutes. We deduce by naturality of the Euler class that

$$
e\left(V_{2}\left(\mathbb{R}^{n+2}\right) \rightarrow \widetilde{G}_{2}\left(\mathbb{R}^{n+2}\right)\right)=j^{*} e\left(\mathbb{S}^{2 n+3} \rightarrow \mathbb{C} \mathbb{P}^{n+1}\right)
$$

and hence, for $n \geq 3$, the Euler class of the bundle $V_{2}\left(\mathbb{R}^{n+2}\right) \rightarrow \widetilde{G}_{2}\left(\mathbb{R}^{n+2}\right)$ is equal to minus one times the preferred generator of $\widetilde{G}_{2}\left(\mathbb{R}^{n+2}\right)$. 
For the low dimensions $n=1$ and $n=2$, the description of the Stiefel manifolds and the corresponding Grassmannians are well known (see for instance Fuks and Rokhlin [12]). The long exact sequence associated with the principal circle bundle $V_{2}\left(\mathbb{R}^{n+2}\right) \rightarrow \widetilde{G}_{2}\left(\mathbb{R}^{n+2}\right)$ gives the numbers for $n=1, N_{e}=2$ and for $n=2, N_{e}=1$.

Associated Hamiltonian action on the cotangent bundle For $u \in \mathbb{S}^{1}$, denote by $\rho(u): V \rightarrow V$ (defined by $\rho(u)(x)=u \cdot x$ ) the action of $\mathbb{S}^{1}$ on $V$. With this circle action on $V$ is associated a $\mathbb{S}^{1}$-action on $T^{*} V$ :

$$
\begin{aligned}
& \mathbb{S}^{1} \times T^{*} V \longrightarrow T^{*} V \\
& (u,(x, \varphi)) \longmapsto(u \cdot x, u \cdot \varphi)
\end{aligned}
$$

where

$$
u \cdot \varphi={ }^{t}\left(T_{x} \rho(u)^{-1}\right)(\varphi),
$$

namely, if $v \in T_{u \cdot x} V$, then

$$
u \cdot \varphi=\varphi\left(T_{x} \rho(u)^{-1} v\right) .
$$

If $T^{*} V$ is endowed with its canonical symplectic structure, this action is Hamiltonian with Hamiltonian function

$$
\begin{aligned}
H: T^{*} V & \longrightarrow \mathbb{R} \\
(x, \varphi) & \longmapsto\langle\varphi, X(x)\rangle
\end{aligned}
$$

(see for instance McDuff and Salamon [15, Exercise 3.12]).

This action is free since the circle action is free on $V$ and the canonical projection $p_{V}: T^{*} V \rightarrow V$ is an equivariant map.

In particular, this action has no fixed point, the associated Hamiltonian vector field $X_{H}$ does not vanish (because the projection of $X_{H}$ on $T V$ by $T p_{V}$ is $X$, a vector field that never vanishes) and all the values of the Hamiltonian are regular.

Symplectic cut of the cotangent bundle A symplectic manifold endowed with a Hamiltonian circle action being given, Lerman [14] has developed a construction which enables one to embed the reduced spaces as codimension 2 symplectic submanifolds of a symplectic manifold (called the symplectic cut)

We describe this construction in the case of the cotangent bundle $T^{*} V$. This symplectic cut is naturally endowed with a Hamiltonian circle action (Proposition 4.5). We are interested in the monotonicity of this symplectic manifold (Section 2) in order to apply a result of Seidel [21] (reminded in Section 4.1) on the periodicity of the Lagrangian Floer homology. 
To define the symplectic cut construction, we consider the following Hamiltonian action of $\mathbb{S}^{1}$ on the product $T^{*} V \times \mathbb{C}$ :

$$
\begin{aligned}
\mathbb{S}^{1} \times\left(T^{*} V \times \mathbb{C}\right) & \longrightarrow T^{*} V \times \mathbb{C} \\
(u,(x, \varphi, z)) & \longmapsto(u \cdot x, u \cdot \varphi, \bar{u} z)
\end{aligned}
$$

with associated Hamiltonian

$$
\begin{aligned}
\tilde{H}: T^{*} V \times \mathbb{C} & \longrightarrow \mathbb{R} \\
(x, \varphi, z) & \longmapsto H(x, \varphi)-\frac{1}{2}|z|^{2} .
\end{aligned}
$$

As for $H$, this action is free and the levels of the Hamiltonian $\tilde{H}$ are regular.

Let $\xi \in \mathbb{R}$ be a (regular) value of $\widetilde{H}$. The level $\widetilde{H}^{-1}(\xi)$ is the disjoint union of

$$
\{(x, \varphi, 0) \mid H(x, \varphi)=\xi\},
$$

diffeomorphic to $H^{-1}(\xi)$, and

$$
\{(x, \varphi, z) \mid H(x, \varphi)>\xi \text { and }|z|=\sqrt{2(H(x, \varphi)-\xi)}\},
$$

diffeomorphic to $H^{-1}((\xi,+\infty)) \times \mathbb{S}^{1}$, the $\mathbb{S}^{1}$-action preserving both sets.

Notice that $\mathbb{S}^{1}$ acts also freely on the level $H^{-1}(\xi)$ since

$$
\begin{aligned}
\tilde{q}_{\xi}: H^{-1}(\xi) & \longrightarrow V \\
(x, \varphi) & \longmapsto x
\end{aligned}
$$

is an equivariant fibration (see Lemma 2.1) and the action is free on the base space.

As the action of $\mathbb{S}^{1}$ on the regular level $\widetilde{H}^{-1}(\xi)$ is free, we can carry out a symplectic reduction. Equipped with the reduced symplectic form, the quotient $W_{\xi}=\tilde{H}^{-1}(\xi) / \mathbb{S}^{1}$ is a symplectic manifold. The decomposition above descends to the quotient, the image of the part $H^{-1}(\xi)$ is a symplectic submanifold $Q_{\xi}$ of $W_{\xi}$ diffeomorphic to $H^{-1}(\xi) / \mathbb{S}^{1}$. Its complement is symplectically diffeomorphic to the open set $H^{-1}((\xi,+\infty))$ (see Figure 1).

Remark 1.2 If $\xi<0$, then $H^{-1}((\xi,+\infty))$ is a neighbourhood of the zero section in the cotangent bundle $T^{*} V$ and this neighbourhood is symplectically embedded as an open submanifold of $W_{\xi}$. In particular, $V$ is canonically embedded as a Lagrangian submanifold of the symplectic cut $W_{\xi}$.

Because of Remark 1.2 and of the monotonicity condition on $W_{\xi}$ (Proposition 2.6), we will deal in the following only with the case $\xi<0$. 


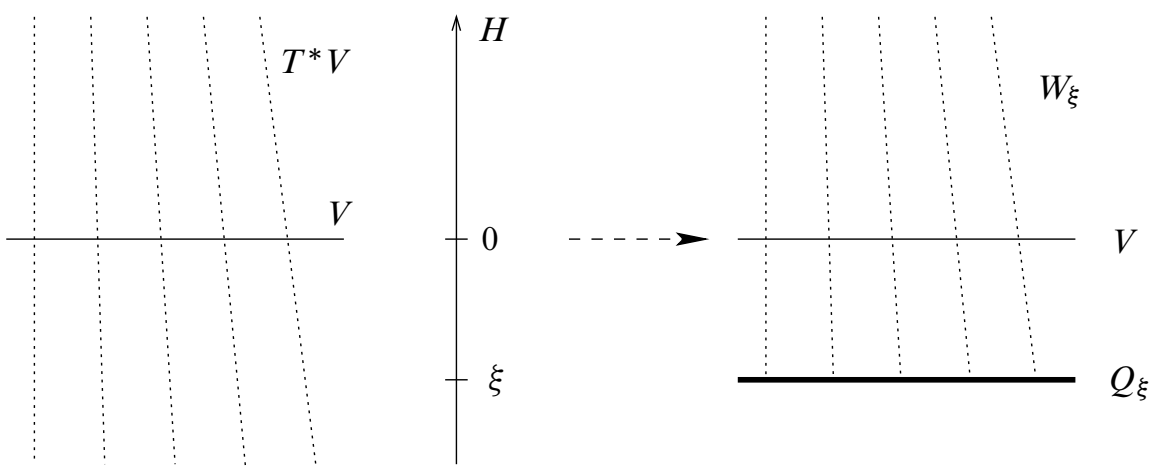

Figure 1: The cotangent bundle (left) and its symplectic cut (right)

Example 0 Let us first study the case of the circle $V=\mathbb{S}^{1}$ acting on itself by multiplication with quotient $B$ reduced to a point. The fundamental field of the action at the point $x$ is then $i x$ (we consider $\mathbb{S}^{1}$ as the unit circle in $\mathbb{C}$ ). The cotangent space $T^{*} \mathbb{S}^{1}$ is diffeomorphic to $\mathbb{S}^{1} \times \mathbb{R}$ : if $x$ is a point of $\mathbb{S}^{1}$ and $\varphi$ is an element of $T_{x}^{*} \mathbb{S}^{1}$, there exists a unique real number $\lambda$ such that $\varphi=\langle\lambda i u, \cdot\rangle$ (denoting by $\langle\cdot, \cdot\rangle$ the scalar product on $\mathbb{C}=\mathbb{R}^{2}$ ). An explicit diffeomorphism from $T^{*} \mathbb{S}^{1}$ to $\mathbb{S}^{1} \times \mathbb{R}$ can be given by mapping the pair $(x, \varphi)$ on the pair $(x, \lambda)$.

The circle action on $T^{*} \mathbb{S}^{1}$ can then be written on $\mathbb{S}^{1} \times \mathbb{R}$ as

$$
u \cdot(x, \lambda)=(u x, \lambda)
$$

and the Hamiltonians become

$$
\begin{gathered}
H(x, \lambda)=H(x, \varphi)=\varphi(i x)=\lambda, \\
\tilde{H}(x, \lambda, z)=H(x, \varphi, z)=\lambda-\frac{1}{2}|z|^{2} .
\end{gathered}
$$

Thus, the level $H^{-1}(\xi)=\left\{(x, \xi) \mid x \in \mathbb{S}^{1}\right\}$ is a circle and the quotient $Q_{\xi}=H^{-1}(\xi) / \mathbb{S}^{1}$ is a point.

For $\tilde{H}$, the level $\widetilde{H}^{-1}(\xi)$ is the set $\left\{\left(x, \xi+\frac{1}{2}|z|^{2}, z\right) \mid x \in \mathbb{S}^{1}, z \in \mathbb{C}\right\}$ and the quotient $W_{\xi}=\widetilde{H}^{-1}(\xi) / \mathbb{S}^{1}$ is symplectomorphic to $\mathbb{C}$. Indeed, the one-to-one map

$$
\begin{aligned}
i_{\mathbb{C}}: \mathbb{C} & \longrightarrow W_{\xi} \\
z & \longmapsto\left[1, \xi+\frac{1}{2}|z|^{2}, z\right]
\end{aligned}
$$


is also onto as each element

$$
\left[x, \xi+\frac{1}{2}|z|^{2}, z\right]=\left[1, \xi+\frac{1}{2}|z|^{2}, x^{-1} z\right]
$$

of $W_{\xi}$ can be written as the image by $i_{\mathbb{C}}$ of a complex number $\left(x^{-1} z=\bar{x} z\right.$ here).

Moreover, the symplectic form $\omega_{\xi}^{W}$ on $W_{\xi}$ is the symplectic reduction of the 2-form $\omega_{\text {can }} \oplus \omega_{\text {std }}$ where $\omega_{\text {can }}$ is the canonical symplectic form on $T^{*} \mathbb{S}^{1}$ and $\omega_{\text {std }}$ is the standard symplectic form on $\mathbb{C}$. But if $\tilde{l}$ is the embedding of $\mathbb{C}$ in $\widetilde{H}^{-1}(\xi)$ defined as

$$
\widetilde{l}(z)=\left(1, \xi+\frac{1}{2}|z|^{2}, z\right)
$$

then

$$
\begin{aligned}
\left(i^{*} \omega_{\xi}^{W}\right)_{z}\left(\zeta_{1}, \zeta_{2}\right) & =\left(\tilde{l}^{*}\left(\omega_{\mathrm{can}} \oplus \omega_{\mathrm{std}}\right)\right)_{z}\left(\zeta_{1}, \zeta_{2}\right) \\
& =\omega_{\mathrm{std}}\left(\zeta_{1}, \zeta_{2}\right)
\end{aligned}
$$

since $z \mapsto\left(1, \xi+\frac{1}{2}|z|^{2}\right)$ takes value in a fibre of the projection $T^{*} \mathbb{S}^{1} \rightarrow \mathbb{S}^{1}$ and the fibres are Lagrangian submanifolds.

The manifold $V=\mathbb{S}^{1}$ is embedded as the zero section in $T^{*} V$ and as the image of the map

$$
x \mapsto[x, 0, \sqrt{-2 \xi}]=[1,0, \bar{x} \sqrt{-2 \xi}]
$$

in $W_{\xi}$ if $\xi<0$. Its image in $\mathbb{C}$ by the symplectic diffeomorphism just described is the circle centered at the origin and of radius $\sqrt{-2 \xi}$. This circle bounds a disc of area $-2 \pi \xi$ (which is also the area of the cylinder that lies between the zero section and the level $\xi$ ) (see Figure 2).

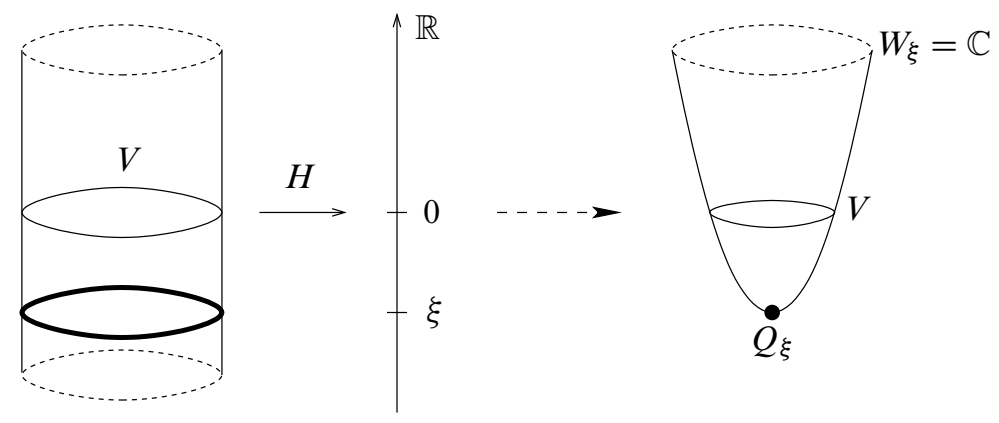

Figure 2: Symplectic cut of $T^{*} \mathbb{S}^{1}$

In the general case of a product $V=B \times \mathbb{S}^{1} \rightarrow B$, there is a symplectic diffeomorphism $T^{*} V \simeq T^{*} B \times T^{*} \mathbb{S}^{1}$. The circle action on $V$ being concentrated on the factor $\mathbb{S}^{1}$, 
the action on $T^{*} V$ is concentrated on $T^{*} \mathbb{S}^{1}$ and the corresponding Hamiltonian on $T^{*} B \times T^{*} \mathbb{S}^{1}$ can be written, for any $((b, \varphi),(x, \lambda)) \in T^{*} B \times T^{*} \mathbb{S}^{1} \simeq T^{*} B \times\left(\mathbb{S}^{1} \times \mathbb{R}\right)$,

$$
H((b, \varphi),(x, \lambda))=\lambda .
$$

Thus, the symplectic cut of $T^{*} V$ (at the level $\xi$ ) is the product of $T^{*} B$ and of the symplectic cut of $T^{*} \mathbb{S}^{1}$ (also at the level $\xi$ ) and hence is symplectomorphic to $T^{*} B \times \mathbb{C}$.

If $\xi$ is negative, $V=B \times \mathbb{S}^{1}$ can be embedded as the product of the zero section of $T^{*} B$ and of the circle centered at the origin of radius $\sqrt{-2 \xi}$.

Let us now analyse the structure of these manifolds. In particular, we would like to know if $W_{\xi}$ is monotone (see Section 2) and in this case, if the zero section is monotone in $W_{\xi}$ (Section 3).

\section{Monotonicity of the symplectic cut}

Recall (see Oh [17] and [18]) that a symplectic manifold $(W, \omega)$ is said to be monotone if there exists a positive constant $K_{W}$ such that for any sphere $w: \mathbb{S}^{2} \rightarrow W$,

$$
\int w^{*} \omega=K_{W} c_{1}(W)([w])
$$

Here and in the following, $c_{1}(W)$ denotes the first Chern class of (the tangent bundle $T W \rightarrow W$ of) $W$. For more general complex vector bundles $E \rightarrow B$, we will use the notation $c_{1}(E \rightarrow B)$ for their first Chern class.

If $W$ is a symplectic manifold, denote by $N_{W}$ its first Chern number, namely the nonnegative generator of the subgroup $\left\langle c_{1}(W), \pi_{2}(W)\right\rangle$ of $\mathbb{Z}$.

We will prove that the symplectic structure and the first Chern class of $W_{\xi}$ can be deduced from those of $Q_{\xi}$. To begin with, let us investigate the reduced manifold $Q_{\xi}=$ $H^{-1}(\xi) / \mathbb{S}^{1}$.

\subsection{The reduced symplectic manifold $Q_{\xi}$}

Lemma 2.1 The projection $\widetilde{q}_{\xi}: H^{-1}(\xi) \rightarrow V$ is an equivariant fibration and descends to the quotients in a fibration

$$
\begin{aligned}
q_{\xi}: Q_{\xi} & \longrightarrow B \\
{[x, \varphi] } & \longmapsto \pi(x) .
\end{aligned}
$$


Proof Let us begin by looking at this projection for $\xi=0$. In this case,

$$
\begin{aligned}
\widetilde{q}_{0}:\{(x, \varphi) \mid\langle\varphi, X(x)\rangle=0\} & \longrightarrow V \\
(x, \varphi) & \longmapsto x
\end{aligned}
$$

is a vector subbundle of the cotangent bundle $T^{*} V \rightarrow V:$ it is the annihilator of the vector field $X$ in the cotangent bundle. Descending to the quotients, $q_{0}$ is also a vector bundle over $B$.

Let $\left\{\alpha_{x}\right\}_{x \in V}$ be a family of linear forms $\alpha_{x} \in T_{x}^{*} V$ such that $\left\langle\alpha_{x}, X(x)\right\rangle=1$ for all $x \in V$ and $\alpha_{u \cdot x}=u \cdot \alpha_{x}$ for all $u \in \mathbb{S}^{1}$. For instance, it can be defined using an $\mathbb{S}^{1}$-invariant Riemannian metric $g$ on $V$ and setting $\alpha_{x}=g_{x}(\cdot, X(x))$. For any $\xi$, this allows to define an equivariant diffeomorphism

$$
\begin{aligned}
\widetilde{\Phi}_{\xi}: H^{-1}(\xi) & \longrightarrow H^{-1}(0) \\
(x, \varphi) & \longmapsto\left(x, \varphi-\xi \alpha_{x}\right)
\end{aligned}
$$

such that $\widetilde{q}_{\xi}=\widetilde{q}_{0} \circ \widetilde{\Phi}_{\xi}$.

Descending to the quotients, the diffeomorphism $\widetilde{\Phi}_{\xi}$ defines a diffeomorphism

$$
\Phi_{\xi}: Q_{\xi} \rightarrow Q_{0}
$$

such that $q_{\xi}=q_{0} \circ \Phi_{\xi}$.

Corollary 2.2 The level $H^{-1}(0)$ (and hence $H^{-1}(\xi)$ as well) has the homotopy type of $V$ and the quotient $Q_{0}$ (and hence $Q_{\xi}$ as well) has the homotopy type of $B$, the homotopy equivalence being given by the projections.

Furthermore, the fibre bundle $q_{0}$ is a particular fibre bundle on $B$ :

Lemma 2.3 The space $Q_{0}$ endowed with the symplectic form obtained by reduction of the canonical symplectic form on $T^{*} V$ is symplectomorphic to the cotangent bundle $T^{*} B$.

A proof of Lemma 2.3 can be found in $\mathrm{Ol}^{\prime}$ shanetskir et al [20, Section 5].

Corollary 2.4 The first Chern class $c_{1}\left(Q_{\xi}\right)$ of $Q_{\xi}$ is zero in $H^{2}\left(Q_{\xi} ; \mathbb{R}\right)$.

Proof Thanks to Lemma 2.3, $Q_{0}$ is symplectomorphic to $T^{*} B$. As the first Chern class $c_{1}\left(T^{*} B\right)$ is a 2-torsion element in $H^{2}\left(T^{*} B ; \mathbb{Z}\right)$ (because the restriction to $B$ of the tangent bundle of $T^{*} B$ is isomorphic to the complexified bundle of a real bundle; see Milnor and Stasheff [16]), $c_{1}\left(Q_{0}\right)$ is 2-torsion element in $H^{2}\left(Q_{0} ; \mathbb{Z}\right)$. 
Moreover, $Q_{\xi}$ varies continuously with $\xi$ and the first Chern class of $Q_{\xi}$ takes values in a discrete space so that $c_{1}\left(Q_{\xi}\right)$ is also a 2-torsion element of $H^{2}\left(Q_{\xi} ; \mathbb{Z}\right)$ and hence vanishes in $H^{2}\left(Q_{\xi} ; \mathbb{R}\right)$.

We determine the class $\left[\omega_{\xi}\right]$ of the symplectic form on $Q_{\xi}$ with the DuistermaatHeckman formula [9] (we follow here the sign conventions of Audin [1]):

Proposition 2.5 The class in $H^{2}\left(Q_{\xi_{0}} ; \mathbb{R}\right)$ of the reduced symplectic form on $Q_{\xi}$ is equal to

$$
\left[\omega_{\xi}\right]=-2 \pi \xi q_{\xi}^{*} e,
$$

still denoting $e$ the Euler class of the circle bundle $V \rightarrow B$.

Proof As all the levels are regular for $H$, by the Duistermaat-Heckman formula, for all $\xi, \xi_{0}$ in $\mathbb{R}$,

$$
\left[\omega_{\xi}\right]-\left[\omega_{\xi_{0}}\right]=\left(\xi-\xi_{0}\right)\left(-2 \pi e\left(H^{-1}(\xi) \rightarrow Q_{\xi}\right)\right)
$$

in $H^{2}\left(Q_{\xi_{0}} ; \mathbb{R}\right)$ where $e\left(H^{-1}(\xi) \rightarrow Q_{\xi}\right)$ is the Euler class of the principal circle bundle $\pi_{Q}: H^{-1}(\xi) \rightarrow Q_{\xi}$.

We now need to compute this Euler class $e\left(H^{-1}(\xi) \rightarrow Q_{\xi}\right)$. As the bundle $H^{-1}(\xi) \rightarrow Q_{\xi}$ is the pullback of the fibre bundle $\pi: V \rightarrow B$ by the projection $q_{\xi}: Q_{\xi} \rightarrow B$,

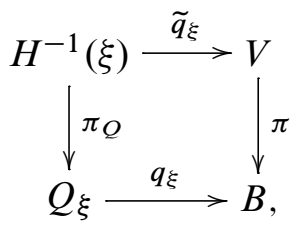

we have by naturality

$$
e\left(H^{-1}(\xi) \rightarrow Q_{\xi}\right)=q_{\xi}^{*}(e(V \rightarrow B))=q_{\xi}^{*} e,
$$

where $q_{\xi}^{*}: H^{2}(B ; \mathbb{Z}) \rightarrow H^{2}\left(Q_{\xi} ; \mathbb{Z}\right)$ is an isomorphism.

Eventually, the Duistermaat-Heckman formula gives

$$
\left[\omega_{\xi}\right]-\left[\omega_{\xi_{0}}\right]=\left(\xi-\xi_{0}\right)\left(-2 \pi q_{\xi}^{*} e\right) .
$$

For $\xi_{0}=0,\left(Q_{0}, \omega_{0}\right)$ is symplectomorphic to the cotangent bundle $\left(T^{*} B, \omega_{\text {can }}\right)$. But $\omega_{\text {can }}$ is an exact form, hence the class $\left[\omega_{\text {can }}\right]$ and a fortiori the class $\left[\omega_{0}\right]$ vanish, so that the Duistermaat-Heckman formula becomes with $\xi_{0}=0$

$$
\left[\omega_{\xi}\right]=-2 \pi \xi q_{\xi}^{*} e .
$$


We could have also used [20, Section 5] and described $Q_{\xi}$ as a magnetic cotangent bundle. Recall that a magnetic cotangent bundle is a cotangent bundle $q: T^{*} B \rightarrow B$ whose symplectic form differs from the canonical symplectic form by a perturbation $q^{*} \sigma$ where $\sigma$ is a closed 2-form on $B$. Here $\sigma=\xi F(\alpha)$ where $F(\alpha)$ is the curvature of a connection 1-form $\alpha$ on the fibre bundle $\pi: V \rightarrow B$.

Example 0 In the case of the trivial bundle, $Q_{\xi}$ is symplectomorphic to $T^{*} B$ for all $\xi$, as the component in $T^{*} B$ of the 1 -form $\alpha$ vanishes. The 2 -form $\omega_{\xi}$ is always exact and $\left[\omega_{\xi}\right]=0$ in $H^{2}\left(Q_{\xi_{0}} ; \mathbb{R}\right)$ as confirmed by Proposition 2.5 .

Examples 1, 2 and 3 In the case of the Hopf bundle for $n \geq 1$ (and similarly in the case of the Stiefel manifolds for $n \geq 3$ ), the class $\left[\omega_{\xi}\right]$ is equal to $-2 \pi$ times the pullback on $T^{*} \mathbb{C} \mathbb{P}^{n}$ of the image in $H^{2}\left(\mathbb{C P}{ }^{n} ; \mathbb{R}\right)$ of the generator of $H^{2}(\mathbb{C P} ; \mathbb{Z})$ (respectively the pullback on $T^{*} \widetilde{G}_{2}\left(\mathbb{R}^{n+2}\right)$ of the image of the generator of $H^{2}\left(\widetilde{G}_{2}\left(\mathbb{R}^{n+2}\right) ; \mathbb{Z}\right)$ ). In the case of example 2 , this class is $-2 \pi p$ times the preferred generator.

\subsection{Consequences for the symplectic cut}

In this paragraph, we want to prove the following:

Proposition 2.6 If $V \rightarrow B$ is a principal circle bundle and if $\xi<0$, then the symplectic cut $W_{\xi}$ is monotone and contains $V$ as a Lagrangian submanifold.

This proposition is a consequence of the bundle structure of $W_{\xi}$ :

Lemma 2.7 The quotient $W_{\xi}$ is the total space of a complex line bundle on $Q_{\xi}$, namely the complex line bundle associated with the principal circle bundle $H^{-1}(\xi) \rightarrow Q_{\xi}$.

Proof By definition, the complex line bundle associated with the principal circle bundle $H^{-1}(\xi) \rightarrow Q_{\xi}$ is $H^{-1}(\xi) \times_{\mathbb{S}^{1}} \mathbb{C} \rightarrow Q_{\xi}$ where $\mathbb{S}^{1}$ acts on $H^{-1}(\xi) \times \mathbb{C}$ by

$$
u \cdot(x, \varphi, z)=(u \cdot x, u \cdot \varphi, \bar{u} z)
$$

and the map

$$
\begin{aligned}
& \widetilde{H}^{-1}(\xi) \longrightarrow H^{-1}(\xi) \times \mathbb{C} \\
& (x, \varphi, z) \longmapsto\left(x, \varphi-\frac{1}{2}|z|^{2} \alpha_{x}, z\right)
\end{aligned}
$$

is an equivariant diffeomorphism for the actions of $\mathbb{S}^{1}$. It descends to the quotients as a diffeomorphism $W_{\xi} \rightarrow H^{-1}(\xi) \times_{\mathbb{S}^{1}} \mathbb{C}$. 
Corollary 2.8 (i) The projection $p_{\xi}: W_{\xi} \rightarrow Q_{\xi}$ is a homotopy equivalence. As a consequence, it is also the case for $q_{\xi} \circ p_{\xi}: W_{\xi} \rightarrow B$.

(ii) The first Chern class of the bundle $p_{\xi}: W_{\xi} \rightarrow Q_{\xi}$ is equal to the Euler class of the circle bundle $H^{-1}(\xi) \rightarrow Q_{\xi}$.

(iii) The bundle $p_{\xi}: W_{\xi} \rightarrow Q_{\xi}$ is the normal bundle of $Q_{\xi}$ in $W_{\xi}$.

Proof of (iii) By Lemma 2.7, $W_{\xi} \rightarrow Q_{\xi}$ is isomorphic to the associated line bundle $H^{-1}(\xi) \times_{\mathbb{S}^{1}} \mathbb{C} \rightarrow Q_{\xi}$. But (see [14, Remark 1.7]), this bundle $H^{-1}(\xi) \times_{\mathbb{S}^{1}} \mathbb{C} \rightarrow Q_{\xi}$ is the normal bundle of the reduced space $Q_{\xi}$ in $W_{\xi}$.

Indeed, we have described a diffeomorphism $H^{-1}(\xi) \times \mathbb{C} \stackrel{\sim}{\rightarrow} \widetilde{H}^{-1}(\xi)$ that induces an isomorphism between the tangent bundles:

$$
T\left(H^{-1}(\xi) \times \mathbb{C}\right) \stackrel{\sim}{\rightarrow} T \tilde{H}^{-1}(\xi) .
$$

Hence, if $j_{\xi}: H^{-1}(\xi) \rightarrow \widetilde{H}^{-1}(\xi)$ denotes the injection of $H^{-1}(\xi)$ in $\widetilde{H}^{-1}(\xi)$, then $j_{\xi}^{*} T\left(H^{-1}(\xi) \times \mathbb{C}\right) \stackrel{\sim}{\rightarrow} j_{\xi}^{*} T \widetilde{H}^{-1}(\xi)$.

But $j_{\xi}^{*} T\left(H^{-1}(\xi) \times \mathbb{C}\right)$ is isomorphic as a bundle on $H^{-1}(\xi)$ to the Whitney sum of $T H^{-1}(\xi)$ and of the trivial bundle $H^{-1}(\xi) \times \mathbb{C}$.

All the maps above are equivariant under the circle action and induce an isomorphism of bundles on $Q_{\xi}$,

$$
i_{\xi}^{*} T W_{\xi} \simeq T Q_{\xi} \oplus\left(H^{-1}(\xi) \times_{\mathbb{S}^{1}} \mathbb{C}\right),
$$

denoting by $i_{\xi}: Q_{\xi} \rightarrow W_{\xi}$ the embedding of $Q_{\xi}$ as the zero section of $W_{\xi}$.

From Corollary 2.8 we get the first Chern class of the tangent bundle $T W_{\xi} \rightarrow W_{\xi}$ :

Lemma 2.9 The first Chern class of $W_{\xi}$ in $H^{2}\left(W_{\xi} ; \mathbb{R}\right)$ is

$$
c_{1}\left(W_{\xi}\right)=\left(q_{\xi} \circ p_{\xi}\right)^{*} e .
$$

Proof By part (iii) of Corollary 2.8,

$$
c_{1}\left(i_{\xi}^{*} T W_{\xi} \rightarrow Q_{\xi}\right)=i_{\xi}^{*} c_{1}\left(T W_{\xi} \rightarrow W_{\xi}\right)=c_{1}\left(T Q_{\xi} \rightarrow Q_{\xi}\right)+c_{1}\left(W_{\xi} \rightarrow Q_{\xi}\right)
$$

and if we apply the inverse isomorphism $p_{\xi}^{*}$ of $i_{\xi}^{*}$,

$$
c_{1}\left(T W_{\xi} \rightarrow W_{\xi}\right)=p_{\xi}^{*}\left(c_{1}\left(T Q_{\xi} \rightarrow Q_{\xi}\right)+c_{1}\left(W_{\xi} \rightarrow Q_{\xi}\right)\right) .
$$


But, by Corollary 2.4, the first Chern class $c_{1}\left(T Q_{\xi} \rightarrow Q_{\xi}\right)$ vanishes in $H^{2}\left(Q_{\xi} ; \mathbb{R}\right)$ and the relation becomes in $H^{2}\left(W_{\xi} ; \mathbb{R}\right)$

$$
\begin{aligned}
c_{1}\left(T W_{\xi} \rightarrow W_{\xi}\right) & =p_{\xi}^{*}\left(c_{1}\left(W_{\xi} \rightarrow Q_{\xi}\right)\right) \\
& =\left(q_{\xi} \circ p_{\xi}\right)^{*} e .
\end{aligned}
$$

Corollary 2.10 The first Chern number $N_{W_{\xi}}$ of $W_{\xi}$ is equal to $N_{e}$, ie, the nonnegative generator of $\left\langle e, \pi_{2}(B)\right\rangle$.

The bundle structure of $W_{\xi} \rightarrow Q_{\xi}$ allows also to determine the class of the symplectic form on $W_{\xi}$ :

Lemma 2.11 If $\omega_{\xi}^{W}$ denotes the symplectic form on $W_{\xi}$ defined by the symplectic reduction, then

$$
\left[\omega_{\xi}^{W}\right]=-2 \pi \xi\left(q_{\xi} \circ p_{\xi}\right)^{*} e
$$

in $H^{2}\left(W_{\xi} ; \mathbb{R}\right)$.

Proof As $Q_{\xi}$ is a symplectic submanifold of $W_{\xi}$, we have

$$
\omega_{\xi}=i_{\xi}^{*} \omega_{\xi}^{W}
$$

(still denoting by $i_{\xi}$ the embedding of $Q_{\xi}$ in $W_{\xi}$ ) and hence

$$
\left[\omega_{\xi}\right]=i_{\xi}^{*}\left[\omega_{\xi}^{W}\right] \text { in } H^{2}\left(Q_{\xi} ; \mathbb{R}\right) .
$$

Applying the inverse isomorphism $p_{\xi}^{*}$ of $i_{\xi}^{*}$, we get

$$
\left[\omega_{\xi}^{W}\right]=p_{\xi}^{*}\left[\omega_{\xi}\right] \text { in } H^{2}\left(W_{\xi} ; \mathbb{R}\right) .
$$

But we have proved that $\left[\omega_{\xi}\right]=-2 \pi \xi q_{\xi}^{*} e$, thus

$$
\left[\omega_{\xi}^{W}\right]=-2 \pi \xi\left(q_{\xi} \circ p_{\xi}\right)^{*} e .
$$

Proof of Proposition 2.6 We can now compare the class of the symplectic form $\left[\omega_{\xi}^{W}\right]$ and the first Chern class $c_{1}\left(W_{\xi}\right)$ in $H^{2}\left(W_{\xi} ; \mathbb{R}\right)$ :

$$
\left[\omega_{\xi}^{W}\right]=-2 \pi \xi c_{1}\left(W_{\xi}\right) .
$$

Together with Remark 1.2, this equality gives the proposition. 
Remark 2.12 It is also possible to carry out the symplectic cutting with the Hamiltonian

$$
(x, \varphi, z) \mapsto H(x, \varphi)+\frac{1}{2}|z|^{2}
$$

instead of $\tilde{H}$. In this case, it is the open subset $H^{-1}(-\infty, \xi)$ that embeds in the new symplectic cut $W_{\xi}^{\prime}$ and the first Chern class is $c_{1}\left(W_{\xi}^{\prime} \rightarrow Q_{\xi}\right)=\left(q_{\xi} \circ p_{\xi}\right)^{*}(-e)$. Thus, it is for $\xi>0$ that $W_{\xi}^{\prime}$ is monotone and contains $V$ as Lagrangian submanifold.

Let us look again at our examples.

Example 0 In the case of the trivial bundle, the Euler class and the class of the symplectic form are trivial in $H^{2}\left(W_{\xi} ; \mathbb{R}\right)$. We already know it, since $W_{\xi}$ is then symplectomorphic to the product of a cotangent bundle and $\mathbb{C}$.

Example 1 In the case of the Hopf bundle, the reduced manifold $W_{\xi}$ is simply connected, with first Chern number $N_{W_{\xi}}=1$. For $\xi<0$, it is monotone and the sphere $\mathbb{S}^{2 n+1}$ can be embedded in $W_{\xi}$ as a Lagrangian submanifold.

Example 2 In the case when $V=L_{p}^{2 n+1}$ is the $(2 n+1)$-dimensional lens space, $W_{\xi}$ is a simply connected symplectic manifold, with first Chern number $N_{W_{\xi}}=p$. It is monotone for $\xi<0$ and the lens space $L_{p}^{2 n+1}$ can be embedded in $W_{\xi}$ as a Lagrangian submanifold.

Example 3 For $n \geq 3, \widetilde{G}_{2}\left(\mathbb{R}^{n+2}\right)$ is simply connected and the Euler class of the bundle $V_{2}\left(\mathbb{R}^{n+2}\right) \rightarrow \widetilde{G}_{2}\left(\mathbb{R}^{n+2}\right)$ is equal to -1 times the generator. The symplectic cut $W_{\xi}$ is hence a simply connected manifold with first Chern number $N_{W_{\xi}}=1$. For $\xi<0, W_{\xi}$ is a monotone symplectic manifold, in which the Stiefel manifold $V_{2}\left(\mathbb{R}^{n+2}\right)$ is embedded as a Lagrangian submanifold.

For the low dimensions $n=1$ and $n=2$, the Grassmannian $\widetilde{G}_{2}\left(\mathbb{R}^{n+2}\right)$ and the symplectic cut are simply connected with Chern numbers for $n=1, N_{W_{\xi}}=2$ and for $n=2, N_{W_{\xi}}=1$.

\section{Monotonicity of the Lagrangian submanifold $V$ in $W$}

We still assume that $V \rightarrow B$ is a principal circle bundle and that $W_{\xi}$ is the symplectic cut of the cotangent bundle of $V$ at the level $\xi$ for a negative real number $\xi$ (in order to simplify the notation, we will omit all the indices $\xi$ in the following). 
Recall (see Oh [17]) that a Lagrangian submanifold $L$ of a symplectic manifold $(W, \omega)$ is monotone if there exist a positive constant $K_{L}$ such that for all $v$ in $\pi_{2}(W, L)$,

$$
\int v^{*} \omega=K_{L} \mu_{L}(v)
$$

where $\mu_{L}: \pi_{2}(W, L) \rightarrow \mathbb{Z}$ denotes the Maslov class of $L$ in $W$ (we will recall the definition of the (relative) Maslov class in the proof of Lemma 3.5).

Moreover, if $L$ is a monotone Lagrangian submanifold of a symplectic manifold $W$, it is known (see [17]) that $W$ must be monotone and that the constants $K_{W}$ and $K_{L}$ satisfy

$$
K_{W}=2 K_{L}
$$

We will also denote by $N_{L}$ the Maslov number of $L$, namely the nonnegative generator of the subgroup $\left\langle\mu_{L}, \pi_{2}(W, L)\right\rangle$ of $\mathbb{Z}$.

Remark 3.1 (i) If an element $v$ of $\pi_{2}(W, L)$ is the image of a class $w$ in $\pi_{2}(W)$, we have the equalities $\mu_{L}(v)=2 c_{1}(W)(w)$ and $\int v^{*} \omega=\int w^{*} \omega$ (see for instance [17]). In particular, $N_{L}$ always divides $2 N_{W}$, (ii) In the case of a simply connected Lagrangian submanifold $L$, the map $\pi_{2}(W) \rightarrow \pi_{2}(W, L)$ is surjective. Consequently, if $W$ is monotone, $L$ is monotone and $N_{L}=2 N_{W}$.

Biran [6] used a remark similar to (ii) for submanifolds $L$ such that $H_{1}(L ; \mathbb{Z})$ is of $q$-torsion (ie for each $\alpha \in H_{1}(L ; \mathbb{Z}), q \alpha=0$ ). Here, we will use the following:

Lemma 3.2 If $L$ is a Lagrangian submanifold of a monotone symplectic manifold $(W, \omega)$ and if $\pi_{1}(L)$ is of $q$-torsion (namely $a^{q}=1$ for any $a$ of $\pi_{1}(L)$ with $q \neq 0$ ), then $L$ is monotone in $W$ and $2 N_{W}$ divides $q N_{L}$.

Proof As $W$ is monotone, there exists a positive constant $K_{W}$ such that for any $w$ in $\pi_{2}(W)$,

$$
\int w^{*} \omega=K_{L} \mu_{L}(v)
$$

Let $v$ be an element of $\pi_{2}(W, L)$. The boundary of $v$ is an element of $\pi_{1}(L)$ and thus if $L$ is of $q$-torsion, $(\partial v)^{q}=\partial\left(v^{q}\right)=1$ in $\pi_{1}(L)$. This means that $v^{q}$ is a sphere $w$ of $W$.

We then have the relations

$$
\begin{aligned}
& 2 c_{1}(w)=\mu_{L}\left(v^{q}\right)=q \mu_{L}(v), \\
& \int w^{*} \omega=\int\left(v^{q}\right)^{*} \omega=q \int v^{*} \omega .
\end{aligned}
$$


The relation (2) implies

$$
q \int v^{*} \omega=2 K_{W} q \mu_{L}(v)
$$

and hence the monotonicity of $L$ in $W$, since $q$ is nonzero.

Moreover by relation (3), for all $v$ in $\pi_{2}(W, L), 2 N_{W}$ divides $q \mu_{L}(v)$ and hence $2 N_{W}$ divides $q N_{L}$.

Remark 3.1 and Lemma 3.2 are useful to study our examples:

Example 0 For the action of $\mathbb{S}^{1}$ on itself by multiplication, we know that

$$
\pi_{2}\left(W, \mathbb{S}^{1}\right)=\pi_{2}\left(\mathbb{C}, \mathbb{S}^{1}\right) \simeq \pi_{1}\left(\mathbb{S}^{1}\right) \simeq \mathbb{Z}
$$

Moreover, the Maslov class of the disc centered at the origin and of radius $\sqrt{-2 \xi}$ (a generator of $\pi_{2}\left(W, \mathbb{S}^{1}\right)$ ) is 2 (it is a disc in $\mathbb{C}$ ) and its area is $-2 \pi \xi$. This means that the area of the disc is equal to $-\pi \xi$ times its Maslov class and that $\mathbb{S}^{1}$ is monotone in $\mathbb{C}$.

Examples 1 and 3 In the case of the Hopf bundle (or of the Stiefel manifolds), as the sphere $V=\mathbb{S}^{2 n+1}$ for $n \geq 1$ (or the Stiefel manifold $V=V_{2}\left(\mathbb{R}^{n+2}\right.$ ) for $n \geq 3$ ) is simply connected, $V$ is monotone in $W$ and $N_{V}=2 N_{W}=2$. These manifolds $V$ are even 2-connected, hence the long exact sequence of the pair $(W, V)$ gives an isomorphism between $\pi_{2}(W, V)$ and $\pi_{2}\left(\mathbb{C P}{ }^{n}\right) \simeq \mathbb{Z}\left(\right.$ or $\left.\pi_{2}\left(\tilde{G}_{2}\left(\mathbb{R}^{n+2}\right)\right) \simeq \mathbb{Z}\right)$.

Example 2 If $V$ is the lens space $L_{p}^{2 n+1}$, its fundamental group is $\mathbb{Z} / p$ and in particular, $\pi_{1}(V)$ is of $p$-torsion. By Lemma 3.2, $V$ is monotone in $W$ and $2 N_{W}$ divides $p N_{V}$, namely $2 p$ divides $p N_{L}$ and hence 2 divides $N_{V}$.

Let us prove that Example 0 essentially describes what happens in general for the Lagrangian submanifold $V$ in the symplectic cut of its cotangent bundle, namely:

Theorem 3.3 Let $\pi: V \rightarrow B$ be a principal circle bundle and let $W$ be the symplectic cut of $T^{*} V$ at the level $\xi$ with $\xi<0$.

Then, $\pi_{2}(W, V)$ is isomorphic to $\mathbb{Z}$, the Lagrangian submanifold $V$ is monotone in $W$ with monotonicity constant $-\pi \xi$ and its Maslov number is 2 .

We begin with the computation of $\pi_{2}(W, V)$ : 
Lemma 3.4 Let $\pi: V \rightarrow B$ be a principal circle bundle and let $W$ be the symplectic cut of $T^{*} V$ at the level $\xi$, with $\xi<0$.

Then $\pi_{2}(W, V)$ is isomorphic to $\mathbb{Z}$, a generator $\phi$ being given by the image of the disc of $\mathbb{C}$ centered at the origin and of radius $\sqrt{-2 \xi}$ in a fibre of the complex line bundle $W \rightarrow Q$.

Proof In order to prove that $\pi_{2}(W, V)$ is isomorphic to $\pi_{1}\left(\mathbb{S}^{1}\right)$, we connect the long exact sequence of the pair $(W, V)$ to the long exact sequence associated with the circle bundle $V \rightarrow B$ :

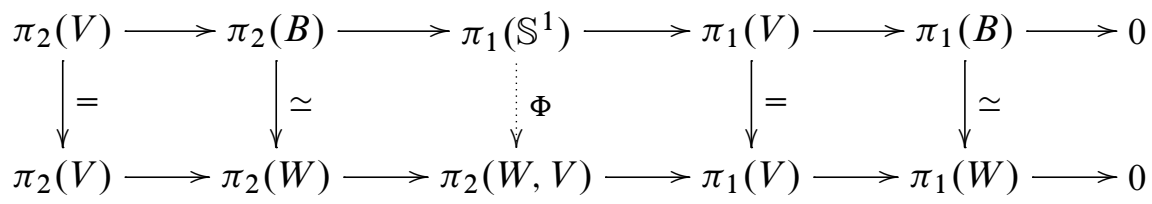

and we define a map $\Phi: \pi_{1}\left(\mathbb{S}^{1}\right) \rightarrow \pi_{2}(W, V)$ that makes the above diagram commute. The map $\Phi$ is given by associating to a generator of $\pi_{1}\left(\mathbb{S}^{1}\right)$ a disk $\phi$, image of the disc of $\mathbb{C}$ centered at the origin and of radius $\sqrt{-2 \xi}$ in a fibre of the complex line bundle $W \rightarrow Q$. (see Appendix A.1 for more details).

By the five-lemma, we conclude that $\Phi$ is an isomorphism (hence, $\pi_{2}(W, V)$ is isomorphic to $\mathbb{Z})$ and that the image of $\pi_{2}(W) \rightarrow \pi_{2}(W, V)$ is equal to the image of $\pi_{2}(B) \rightarrow \pi_{1}\left(\mathbb{S}^{1}\right)$, namely $N_{e} \mathbb{Z}=\left\langle e, \pi_{2}(B)\right\rangle$.

We can determine the Maslov number of $V$ in $W$ thanks to the commutative diagram

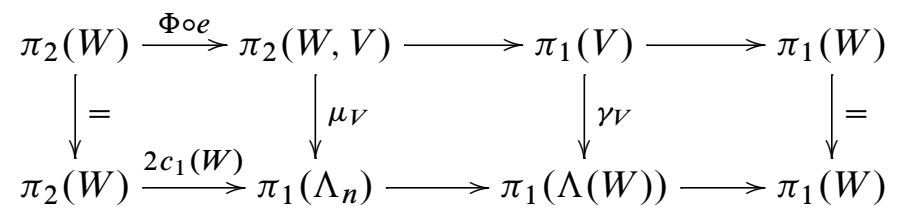

where $\Lambda_{n}$ (for $n$ half the dimension of $W$ ) is the Grassmannian of the Lagrangian subspaces of $\mathbb{R}^{2 n}$ and $\Lambda(W)$ is the fibre bundle associated to the vector bundle $T W \rightarrow W$ with typical fibre $\Lambda_{n}$.

We have $\mu \circ(\Phi \circ e)=2 c_{1}(W)$ and hence if the Euler class $e$ does not vanish on $\pi_{2}(B)$ (in particular, $\pi_{2}(B)$ cannot be trivial), then $\mu_{V}: \pi_{2}(W, V) \simeq \mathbb{Z} \rightarrow \pi_{1}\left(\Lambda_{n}\right) \simeq \mathbb{Z}$ is the multiplication by 2 or -2 and the Maslov number of $V$ is $N_{V}=2$.

In particular, if $e$ is not trivial on $\pi_{2}(B)$, we can say that the Maslov class of the generator of $\pi_{2}(W, V)$ is 2 or -2 . Unfortunately, if we want to conclude for the monotonicity of $V$, we must find out the sign of this class. We use one more time Example 0. 
Lemma 3.5 The Maslov class in $W$ of the generator $\phi$ is 2 .

Proof By definition, we get the Maslov class $\mu_{V}^{W}$ of a disc $v:\left(D^{2}, \mathbb{S}^{1}\right) \rightarrow(W, V)$ by trivialising the tangent bundle $T W$ on the disc:

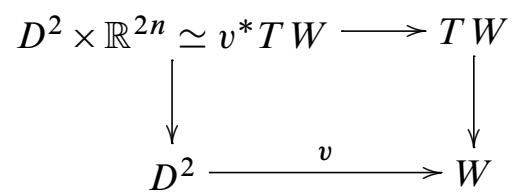

As the restriction $\left.v\right|_{\mathbb{S}^{1}}$ takes its values in $V,\left(\left.v\right|_{\mathbb{S}^{1}}\right)^{*} T V$ defines a loop in $T V$ and after trivialisation of $v^{*} T W$, a loop in the Grassmannian $\Lambda_{n}$ of the linear Lagrangian subspaces of $\mathbb{R}^{2 n}$. The class $\mu_{V}(v)$ is then the (ordinary) Maslov class of this loop of $\Lambda_{n}$.

In our case, the disc $\phi$ lies in the fibre of $W \rightarrow Q$. We describe $T W$ in the neighbourhood of this fibre. Let $\mathcal{U}$ be a contractile neighbourhood of $b_{0}$ in $B$ so that the bundle $V \rightarrow B$ can be trivialised on $\mathcal{U}$ :

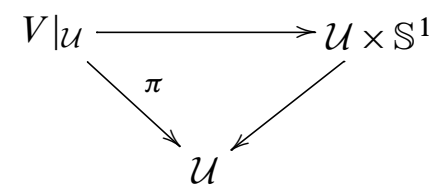

On the open subset $\left.V\right|_{\mathcal{U}}$ of $V$, the restriction of $\pi$ is a trivial bundle. We are therefore in the case of Example 0. This means that $\left.W\right|_{\mathcal{U}}\left(=(p \circ q)^{-1}(\mathcal{U})\right)$ is symplectomorphic to $T^{*} \mathcal{U} \times \mathbb{C}$ and $\left.V\right|_{\mathcal{U}}$ is embedded in $T^{*} \mathcal{U} \times \mathbb{C}$ as the product of the zero section of $T^{*} \mathcal{U}$ and of the circle centered at the origin and of radius $\sqrt{-2 \xi}$. The Maslov class of the disc $\phi$ is hence equal to the Maslov class of the loop $u \mapsto \sqrt{-2 \xi} u$ in $\mathbb{C}$, namely 2 .

Proof of Theorem 3.3 Thanks to the symplectomorphism between $\mathbb{C}$ and the fibre (see Appendix A.1), we also know the area of the disc $\phi$ : it is equal to the area of the disc of $\mathbb{C}$ with center at the origin and radius $\sqrt{-2 \xi}$, namely $-2 \pi \xi$. As in the case of $T^{*} \mathbb{S}^{1}$,

$$
\int v^{*} \omega=-\pi \xi \mu_{V}^{W}(v)
$$

if $v$ is the disc $\phi$ and thus for all $v$ of $\pi_{2}(W, V)$.

In the next section, we are interested in the other monotone Lagrangian embeddings in a symplectic cut. 


\section{Monotone Lagrangian submanifolds in symplectic cuts}

As announced in Introduction, we will apply periodicity results of Seidel [21] to Lagrangian submanifolds in the symplectic cut. We recall here some general results on this periodicity.

\subsection{Seidel's periodicity theorem}

To begin with, let us state the theorem of Seidel [21] (see also Audin [2]):

Theorem 4.1 (Seidel) Let $(W, \omega)$ be a monotone geometrically bounded symplectic manifold endowed with a Hamiltonian circle action. Let $w$ be the sum of the weights of the linearised action at a fixed point.

Assume that there exists a positive integer $N$ such that $2 c_{1}(W)$ is mapped onto zero in $H^{2}(W ; \mathbb{Z} / N \mathbb{Z})$.

Let $L$ be a monotone Lagrangian submanifold of $W$ (with $N_{L} \geq 2$ ) such that the modulo $N$ Maslov class $\left(\gamma_{L}^{W}\right)^{*}\left(\mu^{N}\right)$ of $L$ vanishes in $H^{1}(L ; \mathbb{Z} / N \mathbb{Z})$.

Then the Floer cohomology of $L$ is (absolutely) graded by $\mathbb{Z} / N \mathbb{Z}$ and is periodic of period $2 w$.

Let us recall how the Gauss map $\gamma_{L}^{W}$ and the reduced Maslov class $\mu^{N}$ are defined . If $(W, \omega)$ is a symplectic manifold, we consider as in Section 3 the bundle $\Lambda(W) \rightarrow W$ associated with the tangent bundle whose fibre at a point is the set of linear Lagrangian subspaces of the tangent space to $W$ at that point. If $L$ is a Lagrangian submanifold of $W$, we denote by $\gamma_{L}^{W}: L \rightarrow \Lambda(W)$ the Gauss map that associates to a point of $L$ the tangent space of $L$ at that point.

If $(W, \omega)$ is a symplectic manifold such that $2 c_{1}(W)=0$ in $H^{2}(W ; \mathbb{Z} / N \mathbb{Z})$ for some integer $N$, there exists (see for example [21]) a Maslov class $\mu^{N}$ in $H^{1}(\Lambda(W) ; \mathbb{Z} / N \mathbb{Z}$ ) that extends the modulo $N$ reduction of the ordinary Maslov class on each fibre of the bundle $\Lambda(W) \rightarrow W$. Note that when $W$ is a cotangent bundle, one can take $N=0$ and $\left(\gamma_{L}^{W}\right)^{*}\left(\mu^{N}\right)$ is then the usual Maslov class of a Lagrangian submanifold $L$ in the cotangent bundle.

Remark 4.2 If $W$ is a simply connected symplectic manifold, the assumption " $2 c_{1}(W)$ is mapped on zero in $H^{2}(W ; \mathbb{Z} / N \mathbb{Z})$ " can be replaced by " $N$ divides $2 N_{W}$ " and the assumption " $\left(\gamma_{L}^{W}\right)^{*}\left(\mu^{N}\right)$ vanishes in $H^{1}(L ; \mathbb{Z} / N \mathbb{Z})$ " by “ $N$ divides $N_{L}$ ". 
We recall now how the weights of a Hamiltonian circle action are defined.

If $(W, \omega)$ is endowed with a Hamiltonian circle action, this action defines a map of $\mathbb{S}^{1}$ in the group of symplectic diffeomorphisms of $W$ :

$$
\begin{aligned}
\rho: \mathbb{S}^{1} & \longrightarrow \operatorname{Sp}(W) \\
u & \longmapsto(x \mapsto u \cdot x)
\end{aligned}
$$

At a fixed point $x$, the linearised actions $T_{x} \rho(u)$ (for $u \in \mathbb{S}^{1}$ ) are endomorphisms of $T_{x} W$ whose matrices in a common diagonalisation basis have the form

$$
\left(\begin{array}{ccc}
u^{m_{1}} & & 0 \\
& \ddots & \\
0 & & u^{m_{n}}
\end{array}\right) .
$$

The $m_{i}$ 's are called the weights of the action.

Example In [21], Seidel applied Theorem 4.1 to the projective space $\mathbb{C P}^{n}$. Together with the following theorem of $\mathrm{Oh}$, he proved that there is no simply connected Lagrangian submanifold in $\mathbb{C P}^{n}$ (for the precise statement, see [21, Theorem 3.1]):

Theorem 4.3 (Oh [19]) Let $L$ be a compact monotone Lagrangian submanifold of a symplectic manifold $(W, \omega)$ such that $N_{L} \geq 2$. Denote by $H F(L, L)$ its Floer cohomology.

(i) If $N_{L} \geq n+2$, then $H F(L, L) \cong \bigoplus_{k} H^{k}(L ; \mathbb{Z} / 2 \mathbb{Z})$.

(ii) If $N_{L}=n+1$, then $H F(L, L) \cong \bigoplus_{k} H^{k}(L ; \mathbb{Z} / 2 \mathbb{Z})$ or $\bigoplus_{k \neq 0, n} H^{k}(L ; \mathbb{Z} / 2 \mathbb{Z})$.

Seidel used the following circle action on the projective space:

$$
\begin{aligned}
\mathbb{S}^{1} \times \mathbb{C P}^{n} & \rightarrow \mathbb{C P}^{n} \\
(u,[z]) & \mapsto\left[u z_{0}, z_{1}, \ldots, z_{n}\right] \\
H([z]) & =\frac{\left|z_{0}\right|^{2}}{\sum_{i=0}^{n}\left|z_{i}\right|^{2}} .
\end{aligned}
$$

of the Hamiltonian

The fixed points are the points of the hyperplane $z_{0}=0$, for which the sum of weights is 1 and the point of homogeneous coordinates $[1,0, \ldots, 0]$ for which the sum of weights is $-n$.

We can notice that, in this example, $N_{W}=n+1$ and that the classes of the sums of weights are equal in $\mathbb{Z} / N_{W} \mathbb{Z}$. Thus, we get for all the fixed points the same period 2 
when the Floer cohomology is graded by $\mathbb{Z} / N \mathbb{Z}$ with $N$ dividing $N_{W}$. This is a general fact:

Proposition 4.4 The class in $\mathbb{Z} / N_{W} \mathbb{Z}$ of the sum of weights at a fixed point does not depend on the fixed point.

This may be well known to specialists. We nevertheless include a proof in Appendix A.2.

In the case of the symplectic cut of the cotangent bundle, we have:

Proposition 4.5 The symplectic cut of the cotangent bundle $W_{\xi}$ is endowed with a Hamiltonian circle action and the sum of the weights of the linearised action at a fixed point is equal to 1 .

Proof The symplectic cut $W_{\xi}$ of the cotangent bundle is endowed with the Hamiltonian circle action

$$
\begin{aligned}
\mathbb{S}^{1} \times W_{\xi} & \longrightarrow W_{\xi} \\
(u,[x, \varphi, z]) & \longmapsto[x, \varphi, u z]
\end{aligned}
$$

of the Hamiltonian

$$
\begin{gathered}
h: W_{\xi} \longrightarrow \mathbb{R} \\
{[x, \varphi, z] \longmapsto \frac{1}{2}|z|^{2} .}
\end{gathered}
$$

As the action of $\mathbb{S}^{1}$ is free on $T^{*} V$, the fixed points of this action are the points such that $z=0$, namely the points lying in $Q_{\xi}$.

At a point $(x, \varphi)$ of $Q_{\xi}$, the action of $\mathbb{S}^{1}$ can be linearised under the decomposition

$$
\begin{gathered}
T_{[x, \varphi, 0]} W_{\xi} \simeq T_{[x, \varphi]} Q_{\xi} \oplus\left(H^{-1}(\xi) \times_{\mathbb{S} 1} \mathbb{C}\right)_{[x, \varphi]} \\
\left(\begin{array}{cc}
\text { id } & 0 \\
0 & u
\end{array}\right) .
\end{gathered}
$$

by

It implies that the sum of weights is 1 .

Remark 4.6 This is (modulo $N_{W_{\xi}}$ ) the smallest nonzero sum of weights we can expect. When the assumptions of Theorem 4.1 are fulfilled, the Floer cohomology of a Lagrangian submanifold is 2-periodic.

We will use Seidel's theorem in the next section in order to get obstructions to the existence of Lagrangian submanifolds into a symplectic cut. To apply Seidel's theorem to the symplectic cut, we have to check the following: 
Proposition 4.7 The symplectic cut is geometrically bounded in the sense of Audin, Lalonde and Polterovich [4].

Proof Recall (see [4]) that a symplectic manifold without boundary $(W, \omega)$ is geometrically bounded if there exists on $W$ an almost complex structure $J$ and a complete Riemannian metric $g$ such that:

(a) $J$ is uniformly tamed by $\omega$, that is, there exist strictly positive constants $\alpha$ and $\beta$ such that for all $X, Y \in T W$,

$$
\begin{aligned}
\omega(X, J X) & \geq \alpha g(X, X), \\
|\omega(X, Y)| & \leq \beta\|X\|_{g}\|Y\|_{g} .
\end{aligned}
$$

(b) There exist an upper bound for the sectional curvature of $(W, g)$ and a strictly positive lower bound for the injectivity radius of $(W, g)$.

We also recall that the cotangent bundle $\left(T^{*} V, \omega_{\text {can }}\right)$ of a closed manifold $V$ is geometrically bounded. To see it, we can choose a metric $g$ on $T^{*} V$ induced by a Riemannian metric on $V$ and an $\omega_{\text {can }}$-tame almost complex structure $J$ homogeneous with respect to uniform dilatations in the fibres. We can choose for example the almost complex structure induced by the Levi-Cività connection (as in Floer [10] and Oh [19]).

The product of $\left(T^{*} V, \omega_{\text {can }}\right)$ with $\left(\mathbb{C}, \omega_{\text {std }}, J_{\text {std }}, g_{\text {std }}\right)$ is then also geometrically bounded.

When $V$ is endowed with a free circle action, one can choose a Riemannian metric on $V$ which is invariant by the action of the circle. The metric on the product $T^{*} V \times \mathbb{C}$ is then also invariant for the induced Hamiltonian action so that we get by restriction the upper bounds and lower bound of (a) and (b) on the level $\widetilde{H}^{-1}(\xi)$. Taking the quotient, the metric and the almost complex structure induced on $W_{\xi}$ satisfy (a) and (b), so that $W_{\xi}$ is geometrically bounded.

\subsection{Simply connected embeddings}

For a monotone Lagrangian embedding of a simply connected manifold in a symplectic cut, we have the following topological obstructions:

Theorem 4.8 Let $B$ be a compact manifold of dimension $d-1$ with $d \geq 2$. Let $e$ be an element of $H^{2}(B ; \mathbb{Z})$ such that $e$ is not trivial on $\pi_{2}(B)$ and $2 e=0$ in $H^{2}(B ; \mathbb{Z} / N \mathbb{Z})$ for an integer $N>2$. Let $V^{d} \rightarrow B^{d-1}$ be the principal circle bundle with Euler class $e$. We denote by $W$ a monotone symplectic cut of $T^{*} V$ (that is, a symplectic cut at a negative level). 
If $N>d+2$ or $N=d+2$ with $d$ odd, there is no compact and simply connected Lagrangian submanifold in $W$.

If $N=d+2$ with $d$ even, the $\mathbb{Z} / 2 \mathbb{Z}$-cohomology groups of a compact and simply connected Lagrangian submanifold in $W$ are isomorphic to those of $\mathbb{C} \mathbb{P}^{d / 2}$.

Proof If $2 e=0$ in $H^{2}(B, \mathbb{Z} / N \mathbb{Z})$ for some integer $N>2, N$ divides $2 N_{e}$, so that $2 N_{e}=2 N_{W}>2$.

Let $L$ be a compact and simply connected Lagrangian submanifold in $W$. By Remark 3.1 (ii), its Maslov number in the symplectic cut $W$ is $N_{L}=2 N_{W}$, and in particular $N_{L} \geq 2$. Applying Theorem 4.1, the Floer homology of $L$ is well defined, graded by $\mathbb{Z} / N \mathbb{Z}$ and 2 -periodic.

If moreover $N \geq d+2$, then $N_{L} \geq d+2$ and we can apply Oh's theorem - the Floer homology of $L$ is isomorphic to the ordinary cohomology of $L$ with coefficient in $\mathbb{Z} / 2 \mathbb{Z}$ :

$$
H F(L, L) \cong \bigoplus_{i} H^{i}(L ; \mathbb{Z} / 2 \mathbb{Z})
$$

This leads to a contradiction with the 2 -periodicity if $N \geq d+3$ (see Figure 3); and if

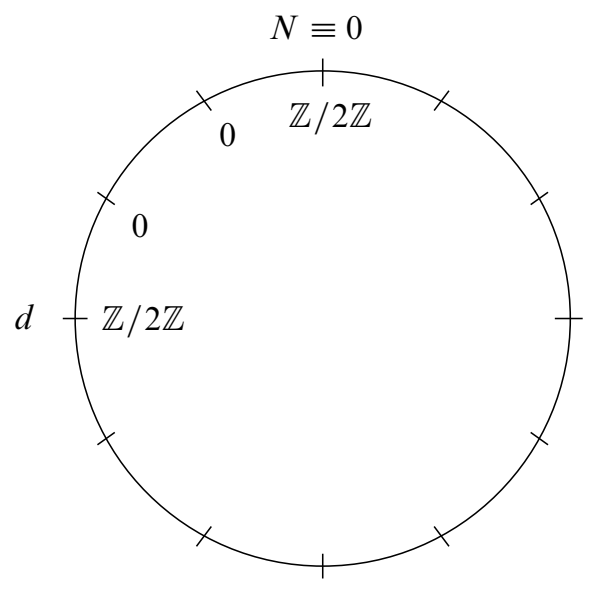

Figure 3: The Floer homology of $L$ when $N_{L} \geq d+2$

$N=d+2$, the periodicity implies that the cohomology groups of $L$ are isomorphic to the cohomology groups of $\mathbb{C P}^{d / 2}$ for even integer $d$ and are trivial if $d$ is odd, which gives another contradiction. 


\subsection{Lagrangian submanifolds of the cotangent bundle}

Let $L$ be a compact Lagrangian submanifold in $T^{*} V$. Then, for $|\xi|$ large enough, it lies in $H^{-1}((\xi ;+\infty))$ and hence can be embedded as a Lagrangian submanifold of the symplectic cut $W_{\xi}$. Such a $\xi$ being now fixed, we will omit the indices $\xi$ in the following.

\subsubsection{Monotonicity of a Lagrangian submanifold of the cotangent bundle into the} symplectic cut In order to understand under which conditions $L$ can be monotone in $W$, we need to know its Maslov class in $W$. In the following, we will relate this class to the Maslov class of $L$ in $T^{*} V$.

Remember that the cotangent bundle $T^{*} V$ of $V$ is a symplectic manifold the first Chern class of which satisfies $2 c_{1}\left(T^{*} V\right)=0$ in $H^{2}\left(T^{*} V ; \mathbb{Z}\right)$. As already noticed in Section 4.1, this implies that there exists a Maslov class $\mu^{T^{*} V} \in H^{1}\left(\Lambda\left(T^{*} V\right) ; \mathbb{Z}\right)$ that extends the ordinary Maslov class on each fibre of $\Lambda\left(T^{*} V\right) \rightarrow T^{*} V$. The Maslov class $\mu_{L}^{T^{*} V} \in H^{1}(L ; \mathbb{Z})$ of a Lagrangian submanifold $L$ in $T^{*} V$ can then be defined as the pullback

$$
\mu_{L}^{T^{*} V}=\left(\gamma_{L}^{T^{*} V}\right)^{*} \mu^{T^{*} V}
$$

The formula connecting the Maslov class of $L$ in $W$ to that of $V$ in $W$ and that of $L$ in $T^{*} V$ is the analogue of the formula given by Lalonde and Sikorav in [13]. We use their notation and denote by $i$ the embedding of $L$ in $T^{*} V$ and $f$ the composition of this embedding and of the projection of $T^{*} V$ on $V$ :

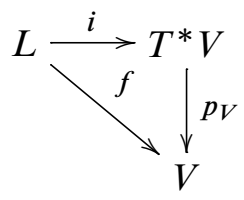

We use the long homotopy exact sequence associated with the triple $\left(W, T^{*} V, L\right)$ :

$$
\pi_{2}\left(T^{*} V, L\right) \longrightarrow \pi_{2}(W, L) \longrightarrow \pi_{2}\left(W, T^{*} V\right) \longrightarrow \pi_{1}\left(T^{*} V, L\right) .
$$

The projection is a homotopy equivalence between $T^{*} V$ and $V$, so that $\pi_{2}\left(W, T^{*} V\right)$ is isomorphic to $\pi_{2}(W, V)$ and the composition of this isomorphism with the map $\pi_{2}(W, L) \rightarrow \pi_{2}\left(W, T^{*} V\right)$ is a map that will be denoted $f_{*}$ (it is actually (id, $\left.f\right)_{*}$ ).

We can now write the formula:

Lemma 4.9 If $\partial: \pi_{2}(W, L) \rightarrow \pi_{1}(L)$ denotes the boundary map, then

$$
\mu_{L}^{W}=\mu_{V}^{W} \circ f_{*}+\mu_{L}^{T^{*} V} \circ \partial .
$$


Proof Let $w:\left(D^{2}, \mathbb{S}^{1}\right) \rightarrow(W, L)$ be a disc with boundary on $L$. Then the map $\pi_{2}(W, L) \rightarrow \pi_{2}\left(W, T^{*} V\right)$ associates to the class of $w$ seen as a disc with boundary in $L$ the class of $w$ seen as a disc with boundary in $T^{*} V$. Thanks to the retraction in the fibres of the projection $p_{V}: T^{*} V \rightarrow V$, we have a homotopy $h$ between $\partial w$ and $p_{V}(\partial w)$ and we can describe the image of the class of $w$ by the isomorphism $\pi_{2}\left(W, T^{*} V\right) \rightarrow \pi_{2}(W, V)$ as the class of the disc $\widetilde{w}=w \# h$ obtained by gluing $w$ and $h$ (see Figure 4), so that $f_{*}[w]=[\tilde{w}]$.

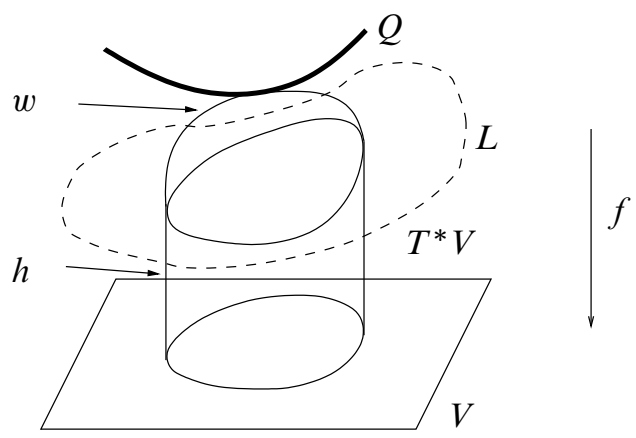

Figure 4: The disc $\widetilde{w}$ obtained by gluing $w$ and $h$

Since $h$ takes value in $T^{*} V$, the difference

$$
\mu_{L}^{W}(w)-\mu_{V}^{W} \circ f_{*}(w)=\mu_{L}^{W}(w)-\mu_{V}^{W}(\tilde{w})
$$

is simply the Maslov class in $T^{*} V$ of the boundary of $h$ (seen as a map of $[0,1] \times[0,1]$ in $T^{*} V$ ) namely the difference between the Maslov classes in $T^{*} V$ of the boundaries of $w$ and of $\widetilde{w}$. And $\tilde{w}$ is a loop in the zero section $V$ of $T^{*} V$, therefore its Maslov class vanishes and we have

$$
\mu_{L}^{W}(w)-\mu_{V}^{W}(\tilde{w})=\mu^{T^{*} V}(\partial w) .
$$

In order to prove the monotonicity of $L$, we need a similar formula for the class of the symplectic form. With the notation of the previous proof, this relation can be written as

$$
\int_{D^{2}} w^{*} \omega_{W}=\int_{D^{2}} \tilde{w}^{*} \omega_{W}+\int_{\mathbb{S}^{1}}(\partial w)^{*} \lambda
$$

where $\lambda$ is the Liouville 1 -form of $T^{*} V$. 
Indeed, we have $\tilde{w}=w \# h$, hence

$$
\begin{aligned}
\int_{D^{2}} \tilde{w}^{*} \omega_{W} & =\int_{D^{2}} w^{*} \omega_{W}+\int_{[0,1] \times[0,1]} h^{*} \omega_{W} \\
& =\int_{D^{2}} w^{*} \omega_{W}+\int_{\mathbb{S}^{1}}\left(p_{V}(\partial w)\right)^{*} \lambda-\int_{\mathbb{S}^{1}}(\partial w)^{*} \lambda .
\end{aligned}
$$

But $p_{V}(\partial w)$ is a loop in $V$, and $V$ is exact in $T^{*} V$, hence the integral $\int_{\mathbb{S} 1}\left(p_{V}(\partial w)\right)^{*} \lambda$ is zero.

Corollary 4.10 Any exact compact Lagrangian submanifold with zero Maslov class in the cotangent bundle of $V$ can be embedded as a monotone Lagrangian submanifold in a symplectic cut of $T^{*} V$ (for some negative level $\xi$ ).

Proof If we restrict to the case of a Lagrangian submanifold $L$ of the cotangent bundle that is exact and with zero Maslov class, the monotonicity of $V$ (Theorem 3.3) implies the monotonicity of $L$ in $W$ as (6) and (7) can then be written: for any disc $w:\left(D^{2}, \mathbb{S}^{1}\right) \rightarrow(W, L)$,

$$
\begin{aligned}
\mu_{L}^{W}([w]) & =\mu_{V}^{W}\left(f_{*}[w]\right)=\mu_{V}^{W}([\tilde{w}]), \\
\int_{D^{2}} w^{*} \omega_{W} & =\int_{D^{2}} \tilde{w}^{*} \omega_{W},
\end{aligned}
$$

which completes the proof.

\subsubsection{Exact compact Lagrangian submanifolds of the cotangent bundle}

Verification of the assumptions of Seidel's periodicity theorem We verify now if Theorem 4.1 can be applied in the symplectic cut to the compact exact Lagrangian submanifolds of the cotangent bundle with vanishing Maslov class.

We have recalled in Section 4.3.1 why we can define a Maslov class

$$
\mu^{T^{*} V} \in H^{1}\left(\Lambda\left(T^{*} V\right) ; \mathbb{Z}\right) .
$$

We will denote by $\left(\mu^{T^{*} V}\right)^{N} \in H^{1}\left(\Lambda\left(T^{*} V\right) ; \mathbb{Z} / N \mathbb{Z}\right)$ the modulo $N$ reduction of $\mu^{T^{*} V}$. As the embedding of $H^{-1}((\xi,+\infty))$ in $W_{\xi}$ is symplectic, we have an embedding

$$
i_{\Lambda}: \Lambda\left(H^{-1}((\xi,+\infty))\right) \rightarrow \Lambda(W)
$$

and if $2 c_{1}\left(W_{\xi}\right)=0$ in $H^{2}\left(W_{\xi} ; \mathbb{Z} / N \mathbb{Z}\right)$ (namely, if $2 e=0$ in $H^{2}(B ; \mathbb{Z} / N \mathbb{Z})$ ), then

$$
\left.\left(\mu^{T^{*} V}\right)^{N}\right|_{\Lambda\left(H^{-1}((\xi,+\infty))\right)}=i_{\Lambda}^{*} \mu^{N} .
$$


Thus, if $L$ is a Lagrangian submanifold of $H^{-1}((\xi,+\infty))$,

$$
\begin{aligned}
\left(\gamma_{L}^{W}\right)^{*} \mu^{N} & =\left(i_{\Lambda} \circ \gamma_{L}^{T^{*} V}\right)^{*} \mu^{N} \\
& =\left(\gamma_{L}^{T^{*} V}\right)^{*}\left(i_{\Lambda}\right)^{*} \mu^{N} \\
& =\left.\left(\gamma_{L}^{T^{*} V}\right)^{*}\left(\mu^{T^{*} V}\right)^{N}\right|_{\Lambda\left(H^{-1}((\xi,+\infty))\right)} .
\end{aligned}
$$

Hence, $\left(\gamma_{L}^{W}\right)^{*} \mu^{N}$ is the modulo $N$ reduction of the Maslov class $\left(\gamma_{L}^{T^{*} V}\right)^{*} \mu^{T^{*} V}$ of $L$ in $T^{*} V$.

In particular, if $L$ is exact, $\left(\gamma_{L}^{W}\right)^{*} \mu^{N}$ is zero in $H^{1}(L ; \mathbb{Z} / N \mathbb{Z})$.

Simply connected Lagrangian submanifolds Simply connected Lagrangian submanifolds are exact, in particular Theorem 4.8 applies, so that we have:

Theorem 4.11 Let $B$ be a compact manifold of dimension $d-1$ with $d \geq 2$. Let $e$ be an element of $H^{2}(B, \mathbb{Z})$ such that $e$ is not trivial on $\pi_{2}(B)$ and $2 e=0$ in $H^{2}(B ; \mathbb{Z} / N \mathbb{Z})$ for some integer $N>2$. Let $V^{d} \rightarrow B^{d-1}$ be a principal circle bundle with Euler class e .

If $N>d+2$ or $N=d+2$ with $d$ odd, there is no compact simply connected Lagrangian submanifold in $T^{*} V$.

If $N=d+2$ with $d$ even, the cohomology groups with coefficients in $\mathbb{Z} / 2 \mathbb{Z}$ of a compact and simply connected Lagrangian submanifold of $T^{*} V$ are isomorphic to those of $\mathbb{C P}^{d / 2}$.

The case $B$ simply connected Lalonde and Sikorav proved in [13] that if $L$ is an exact Lagrangian submanifold of $T^{*} V$, then the index of $f_{*}\left(\pi_{1}(L)\right)$ in $\pi_{1}(V)$ is finite. In the case $B$ (and hence $W$ ) is simply connected, Seidel's theorem (for its simplified statement, see Remark 4.2) gives:

Theorem 4.12 Let $V^{d} \rightarrow B^{d-1}$ be a principal circle bundle with base a compact and simply connected manifold $B$ and with nonzero Euler class. Let $L$ be an exact compact Lagrangian submanifold in $T^{*} V$. We assume that its Maslov class in the cotangent bundle is zero.

Then, if $m$ denotes the index of $f_{*}\left(\pi_{1}(L)\right)$ in $\pi_{1}(V)$,

(i) $m$ divides $N_{e}$ where $N_{e}$ the nonnegative generator of $\left\langle e, \pi_{2}(B)\right\rangle \subset \mathbb{Z}$;

(ii) $2 m \leq d+2$.

For the proof of Theorem 4.12, notice first that: 
Remarks 4.13 (1) Let $V^{d} \rightarrow B^{d-1}$ be a circle bundle with base a compact and simply connected manifold $B$.

If $N_{e}$ is the nonnegative generator of the subgroup $\left\langle e, \pi_{2}(B)\right\rangle$ of $\mathbb{Z}$, then

$$
\pi_{1}(V)=\mathbb{Z} / N_{e} \mathbb{Z} .
$$

In particular, $\pi_{1}(V)$ is cyclic. (2) If $L$ is a compact exact Lagrangian submanifold of $T^{*} V$ with $B$ simply connected and if $m$ is the index of $f_{*}\left(\pi_{1}(L)\right)$ in $\pi_{1}(V)$, then the quotient

$$
\pi_{1}(V) / f_{*}\left(\pi_{1}(L)\right) \cong \mathbb{Z} / m \mathbb{Z}
$$

is also cyclic.

To see (1), one can compute $\pi_{1}(V)$ with the long exact sequence associated to the fibre bundle $V \rightarrow B$ :

$$
\pi_{2}(B) \longrightarrow \pi_{1}\left(\mathbb{S}^{1}\right) \longrightarrow \pi_{1}(V) \longrightarrow \pi_{1}(B)=\{0\} .
$$

As the image of the map $\pi_{2}(B) \rightarrow \pi_{1}\left(\mathbb{S}^{1}\right)$ is $N_{e} \mathbb{Z}, \pi_{1}(V) \cong \mathbb{Z} / N_{e} \mathbb{Z}$ (so that we could have seen in this case that $V$ is monotone in $W$ thanks to Lemma 3.2).

Proof of Theorem 4.12 By Formula (8), $\mu_{L}^{W}=\mu_{V}^{W} \circ f_{*}$.

As the image of $f_{*}: \pi_{2}(W, L) \rightarrow \pi_{2}\left(W, T^{*} V\right)$ is $m \mathbb{Z}$ (because the index of $f_{*}\left(\pi_{1}(L)\right)$ in $\pi_{1}(V)$ is $\left.m\right)$ and $\mu_{V}^{W}$ is the multiplication by 2 , the Maslov number of $L$ is $N_{L}=2 m$.

We also know that $N_{L}$ divides $2 N_{W}=2 N_{e}$ (Remark 3.1), so $m$ necessarily divides $N_{e}$ and we have (i).

Eventually, if $N_{L} \geq d+2$, then first, $N_{L} \geq 2$ so that we can consider the Floer homology of $L$ and secondly the assumptions of Oh's Theorem (Theorem 4.3) are fulfilled, so that the Floer homology is isomorphic to the cohomology of the submanifold:

$$
H F(L, L) \cong \bigoplus_{i} H^{i}(L ; \mathbb{Z} / 2 \mathbb{Z})
$$

By Theorem 4.1 and Proposition 4.5, as $W$ is simply connected and as $2 m$ divides $N_{L}$ and $2 N_{W}$, the Floer homology of $L$ is graded by $\mathbb{Z} / 2 m \mathbb{Z}$,

$$
H F^{i}(L, L)=H^{i}(L ; \mathbb{Z} / 2 \mathbb{Z}),
$$

and by Proposition 4.5 , must be 2 -periodic. In the case when $N_{L} \geq d+3$, this periodicity is in contradiction with Oh's theorem. 
Corollary 4.14 Under the assumptions of Theorem 4.12, if $2 N_{e}>d+2$, then $H^{1}\left(L ; \mathbb{Z} / N_{e} \mathbb{Z}\right) \neq\{0\}$. In particular, $L$ cannot be simply connected (conclusion already contained in Theorem 4.11).

Proof If $2 N_{e}>d+2$, as $2 m \leq d+2$ by Theorem 4.12, the index $m$ of $f_{*}\left(\pi_{1}(L)\right)$ in $\pi_{1}(V)$ is strictly smaller than the cardinal $N_{e}$ of the group $\pi_{1}(V)$. Hence, the subgroup $f_{*}\left(\pi_{1}(L)\right)$ is not trivial, $f_{*}$ is a nonzero homomorphism from $\pi_{1}(L)$ to $\mathbb{Z} / N_{e} \mathbb{Z}$ and $H^{1}\left(L ; \mathbb{Z} / N_{e} \mathbb{Z}\right) \neq\{0\}$.

Applied to the lens spaces, Theorem 4.12 and Corollary 4.14 give the following:

Proposition 4.15 If $L$ is a compact exact Lagrangian submanifold with zero Maslov class in the cotangent bundle of a lens space $L_{p}^{2 n+1}$, then the quotient $\pi_{1}(V) / f_{*}\left(\pi_{1}(L)\right)$ is cyclic and its cardinal, namely the index of $f_{*}\left(\pi_{1}(L)\right)$ in $\pi_{1}(V)$, is less than or equal to $n+1$ and divides $p$.

In particular, if $p$ is a prime number strictly greater than $n+1$, then this index equals 1 .

Proof In the case of the lens spaces $V=L_{p}^{2 n+1}, B=\mathbb{C P} \mathbb{P}^{n}$ is simply connected and $N_{e}=p$. The conclusion of Theorem 4.12 can be written: $m$ divides $p$ and $2 m \leq 2 n+3$. Because of the parity, the case $2 m=2 n+3$ cannot be realised, and finally we have $m \leq n+1$.

In particular, if $p$ is a prime, $m$ is equal to 1 or $p$ and if $p>n+1$, then necessarily $m=1$.

Corollary 4.16 Let $L$ be a Lagrangian submanifold in the cotangent bundle of a lens space $L_{p}^{2 n+1}$ for a integer $p$ such that $p>n+1$. Then $H^{1}(L ; \mathbb{Z} / p \mathbb{Z}) \neq\{0\}$. In particular, $L$ cannot be simply connected nor with finite fundamental group of cardinal $q$ with $\operatorname{gcd}(q, p)=1$

But in the case the fundamental group of $V$ is cyclic, the following can be directly proved without using the symplectic cut of the cotangent bundle:

Theorem 4.17 Let $V^{d} \rightarrow B^{d-1}$ be a circle bundle with basis a compact simply connected manifold $B$. Let $L$ be a compact exact Lagrangian submanifold of $T^{*} V$.

Then the map induced by $f$,

$$
f_{*}: \pi_{1}(L) \longrightarrow \pi_{1}(V),
$$

is surjective. 
Proof By Remarks $4.13(1), \pi_{1}(V)=\mathbb{Z} / N_{e} \mathbb{Z}$ is a quotient of $\pi_{1}\left(\mathbb{S}^{1}\right)=\mathbb{Z}$.

To any abelian subgroup $m \mathbb{Z} / N_{e} \mathbb{Z} \subset \mathbb{Z} / N_{e} \mathbb{Z}$ ( $m$ a divisor of $N_{e}$ ) is associated a finite cyclic covering with $m$ sheets $\tilde{V} \rightarrow V$. The restriction of this covering to each fibre of the bundle $V \rightarrow B$ is just the path-connected covering with $m$ sheets of $\mathbb{S}^{1}$. In particular, if we lift the circle action $\rho(u)$ on $V$ (for $u \in \mathbb{S}^{1}$ ) in a flow $\tilde{\rho}(u)$ on $\widetilde{V}$ (for $u \in \mathbb{R}$ ), then $\widetilde{\rho}(1)$, the flow at time 1 , is a generator of the covering group of $\widetilde{V} \rightarrow V$. If we consider the associated covering $T^{*} \tilde{V} \rightarrow T^{*} V$, the generator of its covering group (denoted by $\chi$ ) is isotopic to the identity through an Hamiltonian isotopy. Now, assume that $j: L \rightarrow T^{*} V$ is an exact Lagrangian embedding such that the image $j_{*}\left(\pi_{1}(L)\right)$ is a subgroup $m \mathbb{Z} / N_{e} \mathbb{Z}$ with $m>1$. This embedding can be lifted to embedding $\widetilde{\jmath}: L \rightarrow T^{*} \widetilde{V}$, and it has the property that $\chi(\widetilde{\jmath}(L)) \cap \widetilde{\jmath}(L)$ is empty. As we know that $\chi$ is Hamiltonian isotopic to the identity, we get a contradiction with the result of Gromov and Floer on the exact Lagrangian submanifolds. Consequently, $m=1$.

Let us consider now the monotone Lagrangian embedding of some particular manifold in the symplectic cut. We begin with the spheres.

\subsection{Monotone Lagrangian embeddings of spheres}

Theorem 4.8 applies of course in the case of an embedding of sphere, but there exists even more obstructions in this case:

Theorem 4.18 Let $B$ be a compact manifold of dimension $d-1$ with $d \geq 2$. Let $e$ be a nonzero element of $H^{2}(B ; \mathbb{Z})$ such that $e$ is not trivial on $\pi_{2}(B)$ and $2 e=0$ in $H^{2}(B ; \mathbb{Z} / N \mathbb{Z})$ for an integer $N>2$. Let $V^{d} \rightarrow B^{d-1}$ be a principal circle bundle with Euler class $e$. We denote by $W$ a monotone symplectic cut of $T^{*} V$.

If $N \geq d+2$ or if $N<d+2$ and $N$ does not divide $d+1$, then the sphere $\mathbb{S}^{d}$ cannot be embedded as a Lagrangian submanifold of $W$, except possibly if $N=4$ and $d \equiv 2[4]$.

Proof As in the proof of Theorem 4.8, $N$ divides $2 N_{e}=2 N_{W}$. As $d \geq 2$, the sphere $L=\mathbb{S}^{d}$ is simply connected and hence exact with Maslov number

$$
N_{L}=2 N_{W}=2 N_{e} \geq 4 .
$$

By Seidel's periodicity Theorem (Theorem 4.1), the Floer homology of $L$ is well defined, graded by $\mathbb{Z} / N \mathbb{Z}$ and 2-periodic.

We then use the following lemma which gives us the Floer homology of the sphere for some values of $N_{W}$. 
Lemma 4.19 Let $W$ be a symplectic monotone manifold of dimension $2 d(d \geq 2)$, with first Chern number $N_{W} \geq 1$, admitting a sphere $\mathbb{S}^{d}$ as Lagrangian submanifold. Then the Floer homology of the sphere $\mathbb{S}^{d}$ is well defined and if $2 N_{W}$ does not divide $d+1$, it is equal to its ordinary cohomology:

$$
H F\left(\mathbb{S}^{d}, \mathbb{S}^{d}\right)=\bigoplus_{k \in \mathbb{Z}} H^{k}\left(\mathbb{S}^{d} ; \mathbb{Z} / 2 \mathbb{Z}\right)
$$

If $N$ does not divide $d+1$, then $2 N_{W}$ cannot divide $d+1$, and by Lemma 4.19 , the Floer homology is isomorphic to the ordinary cohomology. This is in contradiction with the 2-periodicity, except in the case $N=4$ and $d \equiv 2$ [4].

Proof of Lemma 4.19 As the sphere $L=\mathbb{S}^{d}$ is simply connected for $d \geq 2$, it is monotone and $N_{L}=2 N_{W} \geq 2$. Thus, the Floer cohomology of $L$ is well defined.

If $N_{L} \geq d+2$, we can directly apply directly Oh's theorem (Theorem 4.3) and the Floer cohomology of the sphere is equal to its ordinary cohomology.

To extend this result in the case $N_{L}<d+2$, we look precisely at the proof of the theorem of $\mathrm{Oh}$.

First, Oh defines a local Floer homology which is isomorphic to the Morse homology of $L$ : given a Darboux neighbourhood $U$ of $L$ and an isotopy $\Phi$ sufficiently close to the identity so that $\Phi_{1}(L)$ lies in $U$, the local Floer homology is defined with the same complex as the "global" Floer homology, but the differential counts only the solutions of Floer's equation that lie in $U$.

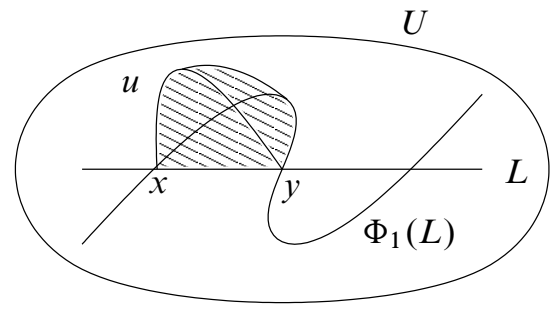

Figure 5: A solution $u$ that does not leave the neighbourhood $U$

We can restrict ourselves to a Hamiltonian isotopy constructed with the help of a Morse function $f: L \rightarrow \mathbb{R}$ on $L:$ as $U$ is symplectomorphic to a neighbourhood of the zero section in $T^{*} L$, we define an Hamiltonian on $U$ by defining it on $T^{*} L$ by the composition of $f$ and the projection $\pi$ of the cotangent bundle:

$$
\begin{aligned}
H: T^{*} L & \rightarrow \mathbb{R} \\
p & \mapsto f(\pi(p))
\end{aligned}
$$


If $f$ is sufficiently $C^{1}$-small, the Hamiltonian isotopy defined by $H$ is sufficiently close to the identity and if $f$ is sufficiently $C^{2}$-small, we can even define an isomorphism between the local Floer homology and the Morse cohomology (see Oh [19] and Floer [10]).

We can then extend $H$ to the whole manifold $W$ by setting it equal to zero out of (a neighbourhood of) $U$. Now, in order to prove the same result for the ("global") Floer homology (and thus the theorem of $\mathrm{Oh}$ ), it remains to see that when $\Phi$ is close enough to the identity ( $f$ is enough $C^{2}$-small), all the solutions of Floer's equation that appear in the definition of the differential (ie the $J$-holomorphic strips between $L$ and $\Phi_{1}(L)$ with Maslov class equal to 1$)$ stay in $U$.

Assume that a solution $u$ "gets out" of $U$.

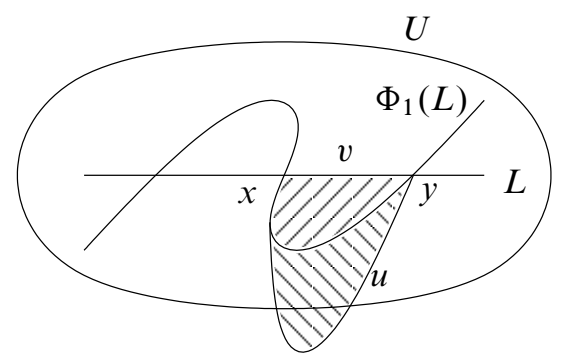

Figure 6: A solution $u$ that leaves $U$

We can glue to $u$ a small strip $v:[0,1] \times[1,2] \rightarrow U \subset W$ in $U$ between $L$ and $\Phi_{1}(L)$ so that we get a disc $w:[0,1] \times[0,2] \rightarrow W$ with boundary on $L$. The fact that the solution $u$ does not stay in $U$ implies (see [19, Proposition 4.1]) that the symplectic area $\int w^{*} \omega$ of $w$ is positive. The monotonicity of $L$ then implies that $\mu_{L}(w)>0$.

But the Maslov classes of the strips satisfy

$$
\mu_{L}(w)=\mu_{u}(x, y)-\mu_{v}(x, y)=1-\mu_{v}(x, y),
$$

and since $v$ is a small strip between $L$ and $\Phi_{1}(L)$, its Maslov index is (see also Viterbo [22])

$$
\mu_{v}(x, y)=\operatorname{ind}_{f}(y)-\operatorname{ind}_{f}(x) \geq-\operatorname{dim}(L),
$$

where $\operatorname{ind}_{f}(x)$ is the Morse index of $x$ for the function $f$.

Hence, $\mu_{L}(w)=\mu_{u}(x, y)-\mu_{v}(x, y)=1-\left(\operatorname{ind}_{f}(y)-\operatorname{ind}_{f}(x)\right) \leq 1+d$.

But $\mu_{L}(w)>0$ and by definition of the Maslov number, $N_{L}$ divides $\mu_{L}(w)$. We get here the (i) of Oh's Theorem: if $N_{L} \geq d+2$, all the solutions stay in $U$ and the Floer homology is the Morse cohomology. 
Here we are interested in the case when $L=\mathbb{S}^{d}$ and we can choose for the Morse function $f$ on $L$ the "height" function which has only two critical points: a minimum of Morse index 0 and a maximum of Morse index $d$.

In this case, in order to have $\mu_{L}(w)>0$ and $N_{L}$ dividing $\mu_{L}(w)$, it is necessary that $\mu_{L}(w)=1+d$.

In consequence, if $N_{L}<d+2$ and $N_{L}$ does not divide $d+1$, all the solutions stay in $U$, and the Floer homology is equal to the local homology, which is isomorphic to the ordinary cohomology.

\subsection{Monotone embeddings of tori}

The case of a monotone Lagrangian torus in a symplectic cut gives one more example of monotone Lagrangian torus with Maslov number equal to 2. This is a result analogous to the result on monotone tori in $\mathbb{C}^{n}$ (see $[8 ; 11]$ ).

Theorem 4.20 Let $V^{d} \rightarrow B^{d-1}$ be a principal circle bundle with basis a compact simply connected manifold $B$ and nonvanishing Euler class. Denote by $W$ a monotone symplectic cut of $T^{*} V$ (that is, a cut at a negative level). Assume that a torus $i: \mathbb{T}^{d} \hookrightarrow W$ is embedded in $W$ as a monotone Lagrangian submanifold with nontrivial Maslov class. Then the Maslov number of $\mathbb{T}$ is equal to 2 .

Proof By Proposition 2.6, $W$ is monotone and its first Chern number is $N_{W}=N_{e}$ where $N_{e}$ is, as before, the Euler number of the circle bundle $V^{d} \rightarrow B^{d-1}$.

The torus being oriented, its Maslov number $N$ is even and greater or equal to 2 . Moreover, as $\mathbb{T}$ is assumed to be monotone in $W$, the Floer homology of the torus $\mathbb{T}$ is well defined. By the periodicity theorem of Seidel (Theorem 4.1), this homology $H F(\mathbb{T}, \mathbb{T})$ is (absolutely) graded by $\mathbb{Z} / N \mathbb{Z}$ and is 2 -periodic for this grading.

Let us assume that $N \geq 3$. We will get a contradiction by studying Oh's spectral sequence [19], and more precisely the description of this spectral sequence given by Biran.

Let $A=\mathbb{Z} / 2 \mathbb{Z}\left[T, T^{-1}\right]$ be the algebra of Laurent polynomials over $\mathbb{Z} / 2 \mathbb{Z}$ in the variable $T$. We define the degree of $T$ to be $N_{L}$. Then

$$
A=\bigoplus_{i \in \mathbb{Z}} A^{i}
$$

where $A^{i}=\mathbb{Z} / 2 \mathbb{Z} T^{i / N_{L}}$ if $i$ is a multiple of $N_{L}$ and $A^{i}=\{0\}$ otherwise. 
Theorem 4.21 (Biran [6]) Let $(M, \omega)$ be a geometrically bounded symplectic manifold and $L$ be a monotone Lagrangian submanifold of $(M, \omega)$ with Maslov number $N_{L} \geq 2$.

There exists a spectral sequence $\left\{E_{r}^{p, q}, d_{r}\right\}$ satisfying the following properties:

(1) For every $r \geq 0, E_{r}^{p, q}$ can be written $E_{r}^{p, q}=V_{r}^{p, q} \otimes A^{p N_{L}}$ with $d_{r}=\delta_{r} \otimes \tau_{r}$, where $\tau_{r}$ is the multiplication by $T^{r}, V_{r}^{p, q}$ are vector spaces over $\mathbb{Z} / 2 \mathbb{Z}$, $\delta_{r}: V_{r}^{p, q} \rightarrow V_{r}^{p+r, q-r+1}$ are homomorphisms and satisfy $\delta_{r} \circ \delta_{r}=0$. Moreover,

$$
V_{r+1}^{p, q}=\frac{\operatorname{ker}\left(\delta_{r}: V_{r}^{p, q} \rightarrow V_{r}^{p+r, q-r+1}\right)}{\operatorname{Im}\left(\delta_{r}: V_{r}^{p-r, q+r-1} \rightarrow V_{r}^{p, q}\right)} .
$$

(2) For $r=1, V_{1}^{p, q}=H^{p+q-p N_{L}}(L ; \mathbb{Z} / 2 \mathbb{Z})$.

(3) $\left\{E_{r}^{p, q}, d_{r}\right\}$ collapses at the $v+1$ step, where $v=\left[(\operatorname{dim}(L)+1) / N_{L}\right]$ and the spectral sequence converges to $H F(L, L)$, that is,

$$
\bigoplus_{p+q=\ell} E_{\infty}^{p, q} \cong H F^{\ell\left(\bmod N_{L}\right)}(L, L) .
$$

(4) For every $p \in \mathbb{Z}$,

$$
\bigoplus_{q \in \mathbb{Z}} E_{\infty}^{p, q} \cong H F(L, L)
$$

Moreover, Buhovski [7] has endowed this spectral sequence of Biran of a multiplicative structure:

Theorem 4.22 (Buhovski [7]) The spectral sequence of Theorem 4.21 can be endowed with a multiplicative structure, that is, for every $r \geq 1$, there exists a product

$$
m_{r}: E_{r}^{p, q} \otimes E_{r}^{p^{\prime}, q^{\prime}} \longrightarrow E_{r}^{p+p^{\prime}, q+q^{\prime}}
$$

such that the differential $d_{r}$ satisfy the Leibniz rule with respect to this product. Moreover, this structure coincide on $E_{1}$ with the usual cup product on the cohomology $H^{*}(L ; \mathbb{Z} / 2 \mathbb{Z})$.

In the case of the torus $\mathbb{T}$ in $W$, the differential at the first page is

$$
\delta_{1}: H^{p+q-p N}(\mathbb{T} ; \mathbb{Z} / 2 \mathbb{Z}) \longrightarrow H^{p+1+q-(p+1) N}(\mathbb{T} ; \mathbb{Z} / 2 \mathbb{Z}) .
$$

In particular, if $p=0$ and $q=1, \delta_{1}: H^{1}(\mathbb{T} ; \mathbb{Z} / 2 \mathbb{Z}) \rightarrow H^{2-N}(\mathbb{T} ; \mathbb{Z} / 2 \mathbb{Z})$ and hence, if $N \geq 3, \delta_{1}$ vanishes on $V_{1}^{0,1}$. The cohomology of the torus is generated as a ring by the elements of $H^{1}(\mathbb{T} ; \mathbb{Z} / 2 \mathbb{Z})$, so that the Leibniz rule for $\delta_{1}$ with respect to the cup product implies that $\delta_{1}$ is zero. 
Consequently, $V_{2}^{p, q}=V_{1}^{p, q}=H^{p+q-p N_{\mathbb{T}}}(\mathbb{T} ; \mathbb{Z} / 2 \mathbb{Z})$. But for $r \geq 2, \delta_{r}$ decreases the degree by $r N-1>3$. We get by induction $\delta_{r}=0$ and

$$
E_{1}=E_{2}=\cdots=E_{r}=E_{\infty}=H F(\mathbb{T}, \mathbb{T}) .
$$

Eventually, $H F(\mathbb{T}, \mathbb{T})=H^{*}(\mathbb{T} ; \mathbb{Z} / 2 \mathbb{Z})$. By Seidel's Theorem, we know that the Floer homology is graded by $\mathbb{Z} / N \mathbb{Z}$ and 2-periodic. This can be written as for every $j \in \mathbb{Z}$,

$$
\bigoplus_{k \in \mathbb{Z}} H^{j+k N}(\mathbb{T} ; \mathbb{Z} / 2 \mathbb{Z})=\bigoplus_{k \in \mathbb{Z}} H^{j+2+k N}(\mathbb{T} ; \mathbb{Z} / 2 \mathbb{Z})
$$

At the dimension level, this equality can be written as for every $j \in \mathbb{Z}$,

$$
\sum_{k \in \mathbb{Z}}\left(\begin{array}{c}
d \\
j+k N
\end{array}\right)=\sum_{k \in \mathbb{Z}}\left(\begin{array}{c}
d \\
j+2+k N
\end{array}\right)
$$

(with the convention $\left(\begin{array}{l}d \\ k\end{array}\right)=0$ if $k \notin\{0, \ldots, d\}$ ).

This means, denoting for $j \in\{0, \ldots, N-1\}$,

that

$$
\begin{gathered}
S_{j}=\sum_{k \in \mathbb{Z}}\left(\begin{array}{c}
d \\
j+k N
\end{array}\right), \\
\left\{\begin{array}{l}
S_{0}=S_{2}=\cdots=S_{N-2}, \\
S_{1}=S_{3}=\cdots=S_{N-1} .
\end{array}\right.
\end{gathered}
$$

As $N$ is even, $N=2 m$, and we sum the $S_{j}$ for the even indexes $j$ :

$$
S_{0}+S_{2}+\cdots+S_{2 m-2}=\sum_{\ell=0, \ell \text { even }}^{d}\left(\begin{array}{l}
d \\
\ell
\end{array}\right)=2^{d-1}
$$

and for the odd indexes $j$ :

$$
S_{1}+S_{3}+\cdots+S_{2 m-1}=\sum_{\ell=0, \ell \text { odd }}^{d}\left(\begin{array}{l}
d \\
\ell
\end{array}\right)=2^{d-1},
$$

and we get that $m S_{0}=m S_{1}=2^{d-1}$. All the $S_{j}$ are thus equal and for every $j$ in $\mathbb{Z}$, $N S_{j}=2^{d}$.

Note that, for a fixed $N$, if the property

$$
\text { for every } j \in \mathbb{Z}, \quad N \sum_{k \in \mathbb{Z}}\left(\begin{array}{c}
d \\
j+k N
\end{array}\right)=2^{d}
$$


is satisfied for $d=d_{0} \geq 2$, then by induction, using Pascal's rule, the property is satisfied for every $d \geq d_{0}$.

In order to get a contradiction, we prove that for every $d_{0} \geq 2$, there exists $d \geq d_{0}$ such that $N S_{0} \neq 2^{d}$. For this purpose, we give another expression of $S_{0}$.

Let $\zeta=\exp (2 i \pi / N)$ be a primitive $N$-root of the unity. Expanding $\left(1+\zeta^{k}\right)^{d}$ for $k \in\{0, \ldots, N-1\}$, and then summing up using the equalities

$$
1+\xi+\xi^{2}+\cdots+\xi^{2 m-1}=0
$$

where $\xi$ is a $N$-root of the unity, we get

$$
\begin{aligned}
N S_{0} & =\sum_{k=0}^{N-1}\left(1+\zeta^{k}\right)^{d} \\
& =2^{d}+\sum_{k=1}^{N-1}\left(1+\zeta^{k}\right)^{d} \\
& =2^{d}+\sum_{k=1}^{N-1} \cos \left(\frac{k d \pi}{N}\right)\left(\cos \left(\frac{k \pi}{N}\right)\right)^{d} .
\end{aligned}
$$

For $d_{0} \geq 2$, choose $d \geq d_{0}$ a multiple of $2 N$. Then $\cos (k d \pi / N)=1$. As for $k \in$ $\{1, \ldots, N-1\}$, we have $0<\pi / N \leq k \pi / N \leq(N-1) \pi / N<\pi / 2$ and $\cos (k \pi / N)>0$. This implies

$$
\begin{gathered}
\sum_{k=1}^{N-1} \cos \left(\frac{k d \pi}{N}\right)\left(\cos \left(\frac{k \pi}{N}\right)\right)^{d}>0 \\
N S_{0}>2^{d}
\end{gathered}
$$

The Floer homology of the torus is thus not 2-periodic and hence $N=2$.

Remark 4.23 We can also prove this result using instead of Biran's spectral sequence endowed with Buhovski's multiplicative structure the dichotomy of Biran and Cornea [5]: the Floer homology of a monotone Lagrangian torus in a symplectic manifold is either trivial or isomorphic to its ordinary cohomology and in the case the Floer homology is trivial, the Maslov number is 2. The proof above gives that the Floer homology cannot be isomorphic to the ordinary cohomology, so that the Floer homology of the torus must be trivial and its Maslov number is 2 . 


\subsection{Monotone embedding of the product of two spheres}

Other examples of manifolds for which the cup product structure is interesting are the product of spheres $\mathbb{S}^{\ell} \times \mathbb{S}^{m}$ :

Theorem 4.24 Let $V^{d} \rightarrow B^{d-1}$ be a principal circle bundle with basis a compact simply connected manifold $B$ and nonvanishing Euler class. Denote by $W$ a monotone symplectic cut of $T^{*} V$. Assume that a product of two spheres $\mathbb{S}^{\ell} \times \mathbb{S}^{m}$ (for $1 \leq \ell \leq m$, $\ell+m=d$ ) can be embedded in $W$ as a monotone Lagrangian submanifold and that its Maslov class is not zero. Then its Maslov number is less then or equal to $m+1$ except when $\ell=1$ and $m=2$ or when $\ell=4$ and $m=6$.

Proof By Künneth Theorem, the ordinary cohomology (with $\mathbb{Z} / 2 \mathbb{Z}$ coefficients) of $L=\mathbb{S}^{\ell} \times \mathbb{S}^{m}$ is, when $\ell<m$,

$$
H^{k}(L ; \mathbb{Z} / 2 \mathbb{Z})= \begin{cases}\mathbb{Z} / 2 \mathbb{Z} & \text { if } k=0, \ell, m, \ell+m, \\ \{0\} & \text { otherwise },\end{cases}
$$

and when $\ell=m$,

$$
H^{k}(L ; \mathbb{Z} / 2 \mathbb{Z})= \begin{cases}\mathbb{Z} / 2 \mathbb{Z} & \text { if } k=0,2 m, \\ \mathbb{Z} / 2 \mathbb{Z} \oplus \mathbb{Z} / 2 \mathbb{Z} & \text { if } k=m, \\ \{0\} & \text { otherwise. }\end{cases}
$$

As in the case of the monotone torus, the Floer homology of $L$ is well defined, absolutely graded by $\mathbb{Z} / N \mathbb{Z}$, if $N$ is the Maslov number of $L$, and is 2 -periodic for this grading. At the first page of the spectral sequence, for $p=0$, the differential

$$
\delta_{1}: H^{q}(\mathbb{T} ; \mathbb{Z} / 2 \mathbb{Z}) \rightarrow H^{q+1-N}(\mathbb{T} ; \mathbb{Z} / 2 \mathbb{Z})
$$

is trivial if $N \geq m+2$, so that $\delta_{1}$ is zero on $V_{1}^{0, q}$ for $q \leq m$. We investigate if $\delta_{1}: V_{1}^{0, q} \rightarrow V_{1}^{1, q}$ can be nonzero for $q=\ell+m$. But $H^{\ell+m}(\mathbb{T} ; \mathbb{Z} / 2 \mathbb{Z})$ is generated by $H^{\ell}(\mathbb{T} ; \mathbb{Z} / 2 \mathbb{Z})$ and $H^{m}(\mathbb{T} ; \mathbb{Z} / 2 \mathbb{Z})$, and as for the torus, the Leibniz rule for $\delta_{1}$ with respect to the cup product implies that $\delta_{1}$ is zero. Analogously, $\delta_{1}$ is zero on every $V_{1}^{p, q}$. Consequently, $V_{2}^{p, q}=V_{1}^{p, q}=H^{p+q-p N_{\mathbb{T}}}(\mathbb{T} ; \mathbb{Z} / 2 \mathbb{Z})$. But for $r \geq 2, \delta_{r}$ decreases the degree by $r N-1>q+1$. We deduce by induction that $\delta_{r}=0$ and

$$
E_{1}=E_{2}=\cdots=E_{r}=E_{\infty}=H F(L, L) .
$$

Eventually, $H F(L, L)=H^{*}(L ; \mathbb{Z} / 2 \mathbb{Z})$. As this Floer homology must be 2-periodic, if $\ell<m$ and $N=m+2$, this is possible only when $m=2$ (and $\ell=1$ ) or when $m=6$ and $\ell=4$.

If $\ell<m$ and $N>m+2$ or if $\ell=m$, the Floer homology cannot be 2-periodic. 


\section{Appendix A Complement to proofs}

\section{A.1 Complement to the proof of Lemma 3.4}

We give here the construction of the map $\Phi: \pi_{1}\left(\mathbb{S}^{1}\right) \rightarrow \pi_{2}(W, V)$ fitting in the following commutative diagram:

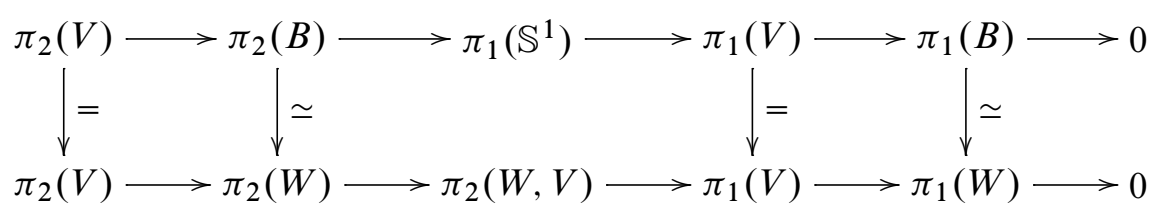

Denote by $x_{0}$ the base point for $\pi_{1}(V), b_{0}$ its projection on $B$ and consider the fibre of $b_{0} \in B \subset Q$ for the complex line bundle $W \rightarrow B$. Adapting the definition of the diffeomorphism between $\mathbb{C}$ and the symplectic cut of $T^{*} \mathbb{S}^{1}$ in Example 0 of Section 1, we can describe a symplectic diffeomorphism between $\mathbb{C}$ and the fibre of $b_{0}$ :

$$
\begin{aligned}
i_{\mathbb{C}}: \mathbb{C} & \longrightarrow W \\
z & \longmapsto\left[x_{0},\left(\xi+\frac{1}{2}|z|^{2}\right) \alpha_{x_{0}}, z\right] .
\end{aligned}
$$

The image of the disc of $\mathbb{C}$ centered at the origin and of radius $\sqrt{-2 \xi}$ is a disc in $W$ with boundary in $V$, since $V$ is embedded in $T^{*} V$ as the zero section and in $W$ by

$$
\begin{aligned}
& V \longrightarrow W \\
& x \longmapsto[x, 0, \sqrt{-2 \xi}],
\end{aligned}
$$

and if $z=\sqrt{-2 \xi} u$ with $u \in \mathbb{S}^{1}$, then $i_{\mathbb{C}}(z)=\left[x_{0}, 0, \sqrt{-2 \xi} u\right]=\left[\bar{u} \cdot x_{0}, 0, \sqrt{-2 \xi}\right]$ lies in the image of $V$ in $W$.

Notice also that the center of the disc is mapped on the image of $b_{0}$ in $W: i_{\mathbb{C}}(0)=$ $\left[x_{0}, \xi \alpha_{x_{0}}, 0\right]$ (indeed, $B$ is embedded in $Q$ by $[x] \mapsto\left[x, \xi \alpha_{x}\right]$ and $Q$ is embedded in $W$ by $[x, \varphi] \mapsto[x, \varphi, 0])$.

In order to define $\Phi$, it is enough to give the image of the generator defined as the class of the loop $t \mapsto e^{-2 i \pi t}$ in $\pi_{1}\left(\mathbb{S}^{1}\right)$. We map this generator on the disc

$$
\begin{aligned}
\phi: D(0, \sqrt{-2 \xi}) & \longrightarrow W \\
z & \longmapsto\left[x_{0},\left(\xi+\frac{1}{2}|z|^{2}\right) \alpha_{x_{0}}, z\right]
\end{aligned}
$$

of $W$ with boundary in $V$ which is the image by $i_{\mathbb{C}}$ of the disc of radius $\sqrt{-2 \xi}$ of $\mathbb{C}$. Let us check that this map $\Phi$ makes the diagram commute. 
The two "side" squares
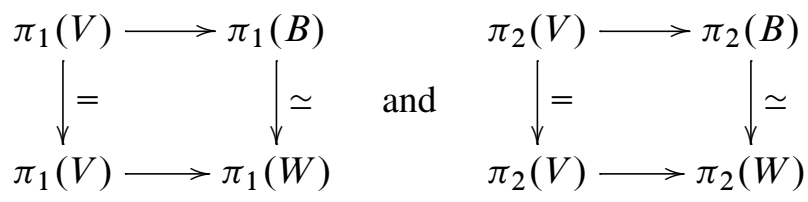

are naturally commutative as the homotopy equivalence between $B$ and $W$ is given by the projection $q_{\xi} \circ p_{\xi}$.

The square

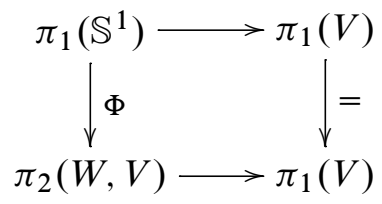

is commutative by construction of $\Phi$. Indeed, the image of the generator $t \mapsto e^{-2 i \pi t}$ of $\pi_{1}\left(\mathbb{S}^{1}\right)$ by the inclusion of $\mathbb{S}^{1}$ in the fibre of $x_{0}$ is $u \mapsto \bar{u} \cdot x_{0}$ and the image of the class of $\phi$ by the boundary map is the class of $u \mapsto\left[x_{0}, 0, \sqrt{-2 \xi} u\right]=\left[\bar{u} \cdot x_{0}, 0, \sqrt{-2 \xi}\right]$ which describes the loop $u \mapsto \bar{u} \cdot x_{0}$ of $V$ in $W$.

Finally, the square

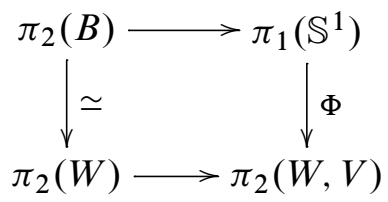

is also commutative. The long exact sequence associated with the bundle $V \rightarrow B$ corresponds to the exact sequence of the pair $\left(V, \mathbb{S}^{1}\right)$ where $\pi_{i}\left(V, \mathbb{S}^{1}\right)$ and $\pi_{i}(B)$ are identified by the isomorphism $\pi_{*}$ and the map $\pi_{i}\left(V, \mathbb{S}^{1}\right) \simeq \pi_{i}(B) \rightarrow \pi_{i-1}\left(\mathbb{S}^{1}\right)$ is the boundary map.

If $w: \mathbb{S}^{2} \rightarrow B$ is a sphere of $B$ with $w(1)=b_{0}$, we can lift it to a disc $v$ of $V$ with boundary in the fibre of $b_{0}($ so $\pi(v)=w)$ such that $v(1)=x_{0}$. The class of $v$ in $\pi_{2}\left(V, \mathbb{S}^{1}\right)$ is then the image of the class of $w$ under the isomorphism $\pi_{2}(B) \stackrel{\simeq}{\rightarrow} \pi_{2}\left(V, \mathbb{S}^{1}\right)$. So the image of the class of $w$ by the map $\pi_{2}(B) \rightarrow \pi_{1}\left(\mathbb{S}^{1}\right)$ is the class of the boundary $\partial v$ of $v$ and its image by the composition $\pi_{2}(B) \rightarrow \pi_{1}\left(\mathbb{S}^{1}\right) \rightarrow \pi_{2}(W, V)$ is the disc $\Phi(\partial v)$ constructed from the loop $\partial v$ of $\mathbb{S}^{1}$ by extending it to a disc in the fibre of $b_{0}$.

On the other hand, the image of the class of a sphere $w$ of $B$ by the map $\pi_{2}(B) \rightarrow \pi_{2}(W)$ is the class of $w$ after the embedding of $B$ in $W$ and the image of the class of a sphere $w$ of $W$ by $\pi_{2}(W) \rightarrow \pi_{2}(W, V)$ is simply the class of $w$ considered as a disc with boundary in $V$. 
In order to verify that the diagram commutes, it is enough to check that in $\pi_{2}(W, V)$, the class of this sphere $w$ is the class of $\Phi(\partial v)$.

As the disk $v$ (the lift of the sphere $w$ of $B$ ) lies in $V$, its class in $\pi_{2}(W, V)$ is trivial. As a consequence, the class in $\pi_{2}(W, V)$ of the disc obtained by sum of $v$ and of $\Phi(\partial v)$ and denoted $v \# \Phi(\partial v)$ is

$$
[v \# \Phi(\partial v)]=[\Phi(\partial v)] .
$$

But $v$ and $\Phi(\partial v)$ have the same boundary, hence $v \# \Phi(\partial v)$ is a sphere representing an element of $\pi_{2}(W)$. In consequence, we already know that the element $[\Phi(\partial v)]$ of $\pi_{2}(W, V)$ is the image of an element of $\pi_{2}(W)$. We still have to prove that $v \# \Phi(\partial v)$ is homotopic to the sphere $w$ in $W$. But for this purpose, it is enough to see that their image by the projection $q_{\xi} \circ p_{\xi}$ (which gives the homotopy equivalence between $W$ and $B$ ) are homotopic in $B$. As $v$ takes value in $V, q_{\xi} \circ p_{\xi}(v)=\pi(v)=w$ and $q_{\xi} \circ p_{\xi}(\Phi(\partial v))=b_{0}$ in $B$ (recall that $\Phi$ is constructed such that the image of a loop is in the fibre of $\left.b_{0}\right)$. Eventually, $q_{\xi} \circ p_{\xi}(v \# \Phi(\partial v))=w \# b_{0}$ which is homotopic to $w$, so that the last square is commutative.

\section{A.2 Proof of Proposition 4.4}

The manifold $W$ being connected, it is enough to check that if $x$ and $y$ are two critical points such that there exists a trajectory of the gradient (of the Hamiltonian $H$ associated with the Hamiltonian circle action) connecting these points, then

$$
w(x) \equiv w(y)\left[N_{W}\right]
$$

where $w(x)$ denotes the sum of weights at the critical point $x$.

If $x$ and $y$ are two such critical points, we can construct a (gradient) sphere in $W$ having these points as poles. We will get the result by studying the relationship between the first Chern class of this sphere and the weights of the action at the poles.

Let $\varphi^{t}$ be a gradient trajectory connecting these two points $x$ and $y$. It means that $\varphi^{t}$ satisfies

$$
\begin{gathered}
\frac{d \varphi^{t}}{d t}=-\operatorname{grad}_{\varphi^{t}} H \\
\lim _{t \rightarrow-\infty} \varphi^{t}=x \quad \text { and } \quad \lim _{t \rightarrow+\infty} \varphi^{t}=y .
\end{gathered}
$$

In order to build the gradient sphere, we consider the trajectories $\varphi_{u}^{t}$ obtained from $\varphi^{t}$ by action of $\mathbb{S}^{1}$, namely if $u \in \mathbb{S}^{1}$, then

$$
\varphi_{u}^{t}: t \mapsto u \cdot \varphi^{t}
$$


Let us prove that, for $u \in \mathbb{S}^{1}, \varphi_{u}^{t}$ is also a trajectory of the gradient.

We have (still denoting by $\rho$ the action)

$$
\begin{aligned}
\frac{d \varphi_{u}^{t}}{d t} & =T_{\varphi^{t}} \rho(u) \frac{d \varphi^{t}}{d t} \\
& =-T_{\varphi^{t}} \rho(u) \operatorname{grad}_{\varphi^{t}} H .
\end{aligned}
$$

To conclude, it is enough to check that $T_{\varphi^{t}} \rho(u) \operatorname{grad}_{\varphi^{t}} H=\operatorname{grad}_{u \cdot \varphi^{t}} H$ or more generally that, for all point $z$ of $W, T_{z} \rho(u) \operatorname{grad}_{z} H=\operatorname{grad}_{u \cdot z} H$. This is true if we choose a metric $g$ on $W$ which is invariant for the action of $\mathbb{S}^{1}$.

We have moreover $\lim _{t \rightarrow-\infty}\left(u \cdot \varphi^{t}\right)=u \cdot\left(\lim _{t \rightarrow-\infty} \varphi^{t}\right)=u \cdot x=x$ (as $x$ is a critical point of $H$, it is a fixed point of the action) and similarly, $\lim _{t \rightarrow+\infty} u \cdot \varphi^{t}=y$.

We can extend the "cylinder without boundary"

$$
\begin{aligned}
\mathbb{S}^{1} \times \mathbb{R} & \rightarrow W \\
(u, t) & \mapsto u \cdot \varphi^{t}
\end{aligned}
$$

to $\mathbb{S}^{1} \times[-\infty,+\infty] \rightarrow W$ by the limits $x$ and $y$ respectively, and then by quotient of the boundaries of the resulting "cylinder with boundary", we get a gradient sphere $w: \mathbb{S}^{2} \rightarrow W$.

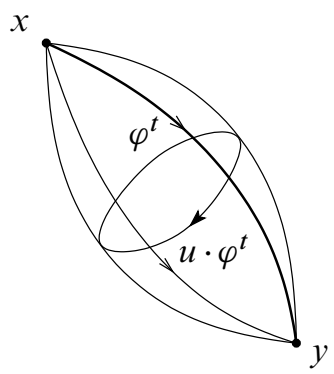

Figure 7: A gradient sphere between $x$ and $y$

In order to get the first Chern class of the sphere $w$, we consider the pullback by $w$ of the determinant bundle on $W$ :

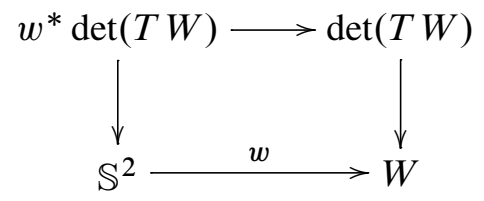


The projection of the tangent bundle is equivariant under the action of $\mathbb{S}^{1}$. It induces an equivariant projection $\operatorname{det}(W) \rightarrow W$.

Moreover, the map defined by

$$
\begin{aligned}
\mathbb{S}^{1} \times \mathbb{R} & \rightarrow W \\
(u, t) & \mapsto u \cdot \varphi^{t}
\end{aligned}
$$

is also equivariant if $\mathbb{S}^{1}$ acts by multiplication on the first factor of $\mathbb{S}^{1} \times \mathbb{R}$. This action can be extended to $\mathbb{S}^{1} \times[-\infty,+\infty] \rightarrow W$ and then descends to the quotient, so that the map $w$ and the projection of the line bundle $f^{*} \operatorname{det}(T W) \rightarrow \mathbb{S}^{2}$ are also equivariant.

But if we decompose the sphere $\mathbb{S}^{2}$ in the union of the two open subset $\mathbb{S}_{+}^{2}$ and $\mathbb{S}_{-}^{2}$ obtained by removing from the sphere respectively the south pole and the north pole, we can trivialise this bundle on each of the two contractile open sets $\mathbb{S}_{+}^{2}$ and $\mathbb{S}_{-}^{2}$ :

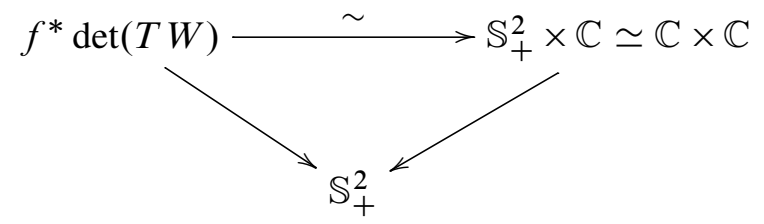

(and the same diagram holds for $\mathbb{S}_{-}^{2}$ ).

If $w(y)$ (respectively $w(x)$ ) denotes the sum of the weights of the linearised action at the point $y$ (respectively at the point $x$ ), the action of $\mathbb{S}^{1}$ on $\mathbb{S}_{+}^{2} \times \mathbb{C} \simeq \mathbb{C} \times \mathbb{C}$ can be written as

$$
u \cdot\left(z_{1}, \zeta_{1}\right)=\left(u z_{1}, u^{w(y)} \zeta_{1}\right)
$$

and on $\mathbb{S}_{-}^{2} \times \mathbb{C} \simeq \mathbb{C} \times \mathbb{C}$,

$$
u \cdot\left(z_{2}, \zeta_{2}\right)=\left(\bar{u} z_{2}, u^{w(x)} \zeta_{2}\right)
$$

Indeed, the linearised action in the chart $\mathbb{C}^{n}$ around $x$ is by definition of the weights $\left(m_{i}(x)\right)_{1 \leq i \leq n}$ at the point $x$ :

$$
u \cdot\left(\begin{array}{c}
v_{1} \\
\vdots \\
v_{n}
\end{array}\right)=\left(\begin{array}{ccc}
u^{m_{1}(x)} & & 0 \\
& \ddots & \\
0 & & u^{m_{n}(x)}
\end{array}\right)\left(\begin{array}{c}
v_{1} \\
\vdots \\
v_{n}
\end{array}\right)
$$

and the determinant of the matrix is $u^{w(x)}$.

We can rebuild the complex line bundle $f^{*} \operatorname{det}(T W) \rightarrow \mathbb{S}^{2}$ by gluing $\mathbb{S}_{+}^{2} \times \mathbb{C}$ and $\mathbb{S}_{-}^{2} \times \mathbb{C}$ on their common boundary $\left|z_{1}\right|=\left|z_{2}\right|=1$. The gluing map can be expressed 
in terms of the first Chern class:

$$
\Phi\left(z_{1}, \zeta_{1}\right)=\left(\bar{z}_{1}, z_{1}^{c_{1}(f)} \zeta_{1}\right)
$$

The action of $\mathbb{S}^{1}$ must be compatible with the gluing, hence

But

$$
\Phi\left(u \cdot\left(z_{1}, \zeta_{1}\right)\right)=u \cdot \Phi\left(z_{1}, \zeta_{1}\right) .
$$

$$
\begin{aligned}
\Phi\left(u \cdot\left(z_{1}, \zeta_{1}\right)\right) & =\Phi\left(u z_{1}, u^{w(y)} \zeta_{1}\right) \\
& =\left(\overline{u z_{1}},\left(u z_{1}\right)^{c_{1}(f)} u^{w(y)} \zeta_{1}\right)
\end{aligned}
$$

and

$$
\begin{aligned}
u \cdot \Phi\left(z_{1}, \zeta_{1}\right) & =u \cdot\left(\bar{z}_{1}, z_{1}^{c_{1}(f)} \zeta_{1}\right) \\
& =\left(\overline{u z}_{1}, u^{w(x)} z_{1}^{c_{1}(f)} \zeta_{1}\right) .
\end{aligned}
$$

Thus, we necessarily have $c_{1}(f)+w(y)=w(x)$ and as $N_{W}$ divides $c_{1}(f)$,

$$
w(x) \equiv w(y)\left[N_{W}\right]
$$

\section{Appendix B Symplectic cut with semifree circle action}

We assume in this section that the action of $\mathbb{S}^{1}$ on $V$ is semifree. It induces as in the case of the free circle action on $V$ a Hamiltonian action on $T^{*} V$ of Hamiltonian

$$
H(x, \varphi)=\langle\varphi, X(x)\rangle .
$$

The fixed points of the induced action on $T^{*} V$ sit in the $0-$ level of $H$. All the other levels of $H$ are regular and the action of $\mathbb{S}^{1}$ on these levels is free. Consequently, the nonzero levels of $\widetilde{H}$ are also regular and we can carry out the symplectic cutting at these levels. This time again, we will be interested in the symplectic cut at a negative level.

In the following, we assume that $\xi$ is a negative real number. We want to know if in this case, the symplectic manifold (still denoted $W_{\xi}$ or $W$ for simplicity) is monotone.

\section{B.1 Sum of weights}

The fixed points of the circle action on the symplectic cut described at Section 4.1 are now not only the points of the symplectic submanifold $Q_{\xi}$ but also the fixed points in $H^{-1}((\xi ;+\infty))$. Moreover, the fixed points for the action of the circle on the symplectic cut on the open set $H^{-1}((\xi ;+\infty))$ are the same as the fixed points for the action of the circle on $T^{*} V$. As we noticed above, these fixed points belong to $H^{-1}(0)$, and hence are embedded in the symplectic cut at the negative level $\xi$. 
In particular, any fixed point of $V$ is after the canonical embedding of $V$ in the symplectic cut a fixed point of the circle action on $W_{\xi}$. We prove that the sum of weights in these points is zero. It is enough to prove this fact for the circle action on the cotangent bundle.

Lemma B.1 The sum of the weights in a fixed point of the zero section $V$ for the action of the circle on the cotangent bundle is zero.

Proof We still denote by $\rho$ the action of $\mathbb{S}^{1}$ on $V$ and we denote by $\tilde{\rho}$ the action induced on $T^{*} V$ :

$$
\begin{aligned}
\mathbb{S}^{1} \times\left(T^{*} V \times \mathbb{C}\right) & \longrightarrow T^{*} V \times \mathbb{C} \\
(u,(x, \varphi, z)) & \longmapsto(u \cdot x, u \cdot \varphi, \bar{u} z) .
\end{aligned}
$$

In a fixed point $x$ of $V$, for every $u \in \mathbb{S}^{1}$,

$$
T_{x} \rho(u): T_{x} V \rightarrow T_{x} V
$$

is an endomorphism of $T_{x} V$. We choose again an invariant metric $g$ on $V$. This means that $T_{x} \rho(u)$ is an isometry for the scalar product on $T_{x} V$ given by $g_{x}$. Denote by $A(u)$ its matrix in an orthonormal basis for the scalar product on $T_{x} V$.

We know that the circle action on $T^{*} V$ is Hamiltonian, in particular symplectic. By definition, the sum of the weights in a fixed point is the sum of the exponents of the variable $u \in \mathbb{S}^{1}$ when we write the matrix of the action at a common basis of diagonalisation. In order to prove that the sum of weights is zero, it is enough to prove that the determinant of this matrix is equal to 1 for every $u$.

We express the circle action matrix on $T^{*} V$ in terms of the action matrix on $V$. On the zero section of the cotangent bundle, the tangent space at $x$ can be decomposed in

$$
T_{(x, 0)}\left(T^{*} V\right) \simeq T_{x} V \oplus T_{x}^{*} V .
$$

In the basis obtained by considering the union of the orthonormal basis on $T_{x} V$ used to define $A(u)$ and its dual basis, the matrix of $T_{x} \widetilde{\rho}(u)$ is

$$
\tilde{A}(u)=\left(\begin{array}{cc}
A(u) & 0 \\
0 & { }^{t} A(u)^{-1}
\end{array}\right)=\left(\begin{array}{cc}
A(u) & 0 \\
0 & A(u)
\end{array}\right) .
$$

Hence the matrix $\widetilde{A}(u)$ is an element of the orthogonal matrices $O(2 d)$ (as $A(u) \in$ $O(d)$ ) and of the symplectic matrices $S p(2 d)$ (as the action is symplectic).

Moreover, we can choose for the almost complex structure $J_{g}$ on the cotangent bundle the one defined thanks to a Levi-Cività connection for the metric on $V$ (as in Floer [10] and Oh [19]). This connection maps the vertical tangent vectors on the horizontal 
tangent vectors. In particular, for the decomposition of the tangent space at $x$ above, if $v \in T_{x} V, J_{g}(v)=g(v, \cdot)$, so that the matrix $\widetilde{A}(u)$ of $O(2 d) \cap S p(2 d) \simeq U(d)$ correspond to the matrix $A(u)$ in $U(d)$.

We already know that $\operatorname{det}(A(u)) \in\{-1 ; 1\}$ as $A(u)$ is orthogonal. Moreover, the map $u \mapsto \operatorname{det}(A(u))$ is continuous and $\operatorname{det}(A(0))=\operatorname{det}(\mathrm{Id})=1$, so that this map is a constant map equal to 1 and the sum of the weights is equal to 0 .

\section{B.2 Monotony of the symplectic cut}

In order to study the symplectic cut in the case of a semifree circle action, we will use the manifold $V_{\text {reg }}$ obtained by taking away of $V$ the fixed points. The circle acts freely on $V_{\text {reg }}$ and we denote by $B_{\text {reg }}=V_{\text {reg }} / \mathbb{S}^{1}$ the quotient by the action.

As the action of the circle is semifree, a consequence of the slice theorem (see for instance Audin [1, Theorem I.2.1]) is that the submanifold consisting of the fixed points is at least of codimension 2 and $V_{\text {reg }}$ (and consequently also $B_{\text {reg }}$ ) is path-connected.

The regular levels $H^{-1}(a)$ of $H$ (for $a \neq 0$ ) are, as in the case of the free circle action, isomorphic to vector bundles over $V_{\text {reg }}$ (they are isomorphic to the vector bundle $\left.\left.H^{-1}(0)\right|_{V_{\text {reg }}} \rightarrow V_{\text {reg }}\right)$, hence are deformation retracts of $V_{\text {reg }}$.

Analogously, the quotients $Q_{a}=H^{-1}(a) / \mathbb{S}^{1}$, for $a \neq 0$, are, as in the free action case, fibre bundle over $B_{\text {reg }}$ isomorphic to $T^{*} B_{\text {reg }}$ and hence have the homotopy type of $B_{\text {reg }}$.

In order to prove that $W=W_{\xi}$ is monotone if $\xi<0$, we study the group $H_{2}(W)$.

Let

$$
\mathcal{U}=H^{-1}((a ;+\infty)) \text { and } \mathcal{V}=H^{-1}((\xi ; b)) \sqcup Q_{\xi}
$$

for $\xi<a<b<0$ (see Figure 8). These are two open sets of $W$ such that $\mathcal{U} \cap \mathcal{V}=$ $H^{-1}((a ; b)), V$ is a retract of $\mathcal{U}$ (thanks to the restriction of the retraction of the cotangent bundle on its zero section), $Q_{\xi}$ is a retract of $\mathcal{V}$ (it is a open disc fibre bundle over $Q_{\xi}$ with projection map the restriction of the one we had for $W \rightarrow Q$ in the case of the free action) and $\mathcal{U} \cap \mathcal{V}$ retracts on a regular level of $H$, thus has the homotopy type of $V_{\text {reg. }}$

We can determine $H_{2}(W)$ by using the Mayer-Vietoris exact sequence for the union $W=\mathcal{U} \cup \mathcal{V}$. The spectral sequence can be written here as

$$
H_{2}(V) \oplus H_{2}\left(B_{\mathrm{reg}}\right) \stackrel{f_{1}}{\longrightarrow} H_{2}(W) \stackrel{f_{2}}{\longrightarrow} H_{1}\left(V_{\mathrm{reg}}\right) \stackrel{f_{3}}{\longrightarrow} H_{1}(V) \oplus H_{1}\left(B_{\mathrm{reg}}\right)
$$

where $f_{3}$ is the map induced by the inclusion of $V_{\text {reg }}$ in $V$ and the projection of $V_{\text {reg }}$ on $B_{\text {reg }}$. 


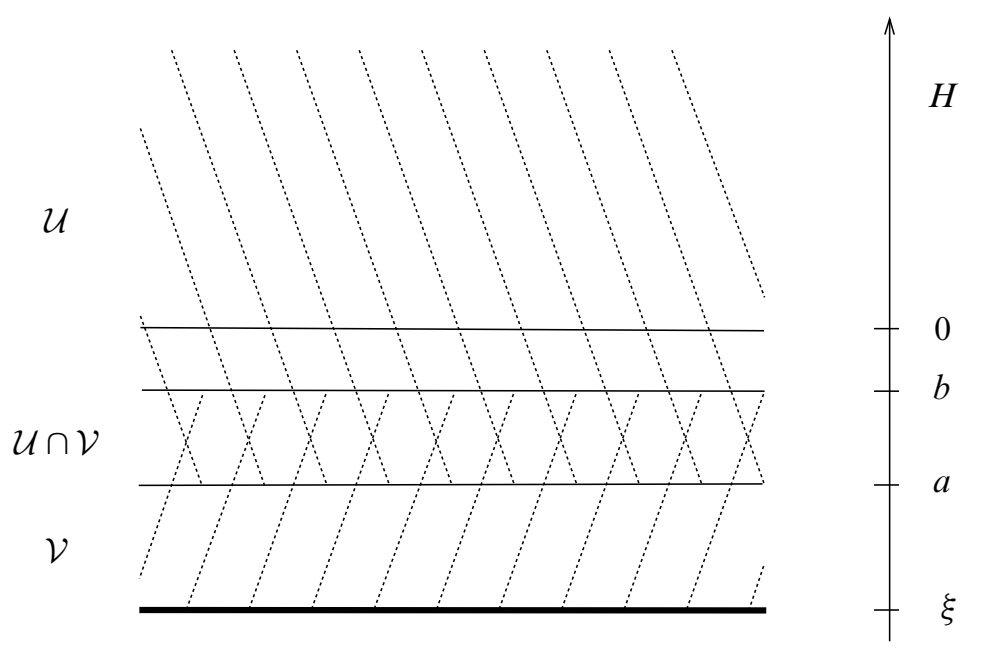

Figure 8: The union $W=\mathcal{U} \cup \mathcal{V}$

If we have a section $s$ of the map

$$
\overline{f_{2}}: H_{2}(W) \longrightarrow \operatorname{ker} f_{3}
$$

induced by $f_{2}$, then by Mayer-Vietoris exact sequence, $H_{2}(W)$ is generated by the image in $H_{2}(W)$ of $H_{2}(V) \oplus H_{2}\left(B_{\text {reg }}\right) \simeq H_{2}(V) \oplus H_{2}(Q)$ by the map $f_{1}$ and by the image of the section $s$.

We describe such a section $s$. We begin with the description of ker $f_{3}$. If $x \in V$ is a regular point in the neighbourhood of a codimension 2 submanifold consisting of fixed points, then the orbit of this point $x$ under the circle action is a 1-dimensional cycle in $V_{\text {reg }}$ whose image in $V$ by the inclusion and image in $B_{\text {reg }}$ by projection are homologous to points.

We then prove that such cycles in the neighbourhood of the codimension 2 fixed points submanifolds generate ker $f_{3}$. On the one hand, by the homotopy long exact sequence for the circle bundle $V_{\text {reg }} \rightarrow B_{\text {reg }}$, the 1-dimensional cycles of $V_{\text {reg }}$ which project on cycles homologous to a point in $B_{\text {reg }}$ are homologous to orbits of points of $V_{\text {reg }}$ for the action of $\mathbb{S}^{1}$. On the other hand, if $V_{\text {neigh }}$ denotes a neighbourhood given by the slice theorem of the fixed points submanifolds of $V$, then the Mayer-Vietoris exact sequence for the union $V=V_{\text {reg }} \cup V_{\text {neigh }}$ gives that the orbit of a point of $V_{\text {reg }}$ under the action of $\mathbb{S}^{1}$ is homologous to a point in $V$ if and only if this orbit is homologous to an orbit in $V_{\text {neigh }}$. 
A section $s$ can then be defined the following way. Let $x_{0}$ be a fixed point of the circle action on $V$. The point $\left[x_{0}, 0, \sqrt{-2 \xi}\right] \in H^{-1}(0)$ is then a critical point of the Hamiltonian $h$ of the circle action on the symplectic cut (see Section 4.1). Let $[x, \varphi, z]$ be a regular point in the unstable manifold of $\left[x_{0}, 0, \sqrt{-2 \xi}\right]$ such that $x$ is in a neighbourhood (given by the slice theorem) of $x_{0}$. We assume that $a$ and $b$ are chosen such that $[x, \varphi, z]$ belongs to $\mathcal{U} \cap \mathcal{V}$.

The trajectory $\gamma_{t}$ of the gradient of $h$ which goes through $[x, \varphi, z]$ at $t=0$ goes to $\left[x_{0}, 0, \sqrt{-2 \xi}\right]$ as $t$ goes to $-\infty$. For $t>0, \gamma_{t}$ lies in the disc bundle $\mathcal{V}$ over $Q$ so that $\gamma_{t}$ goes, as $t$ goes to $+\infty$, to a fixed point $q$ which belongs to $Q$. Letting the circle act on this gradient trajectory, we get a gradient sphere with north pole $x_{0}$, south pole $q$, whose "equator" $\mathbb{S}^{1} \cdot \gamma_{0}$ projects over $\mathbb{S}^{1} \cdot x$ in $V_{\text {reg }}$ which is a loop around the fixed point $x_{0}$. This means that we can map any cycle around a codimension 2 fixed points submanifold of $V$ to a gradient sphere in $W$ and this defines a section $s$ of $\bar{f}_{2}$. Now, it is enough, in order to establish the monotonicity of $W$ to verify that the monotonicity relation is satisfy on the images of $\mathrm{H}_{2}(\mathcal{U}), \mathrm{H}_{2}(\mathcal{V})$ and on the gradient spheres in the image of the section $s$.

(1) The set $\mathcal{U}$ is an open subset of $T^{*} V$ which is symplectically embedded in $W$. For any element $c$ of $H_{2}(\mathcal{U}), c_{1}(W)(c)=0$ and $[\omega](c)=0$ (the form is exact on this open set).

(2) If we proceed to the symplectic cutting construction $W_{\text {reg }}$ at the level $\xi$ for the principal circle bundle $V_{\text {reg }} \rightarrow B_{\text {reg }}, \mathcal{V}$ is a disc bundle sitting inside the line bundle $W_{\text {reg }} \rightarrow Q_{\xi}$. We have proved that in the case of a free action we have in $H^{2}\left(W_{\text {reg }} ; \mathbb{R}\right)$,

$$
[\omega](c)=-2 \pi \xi c_{1}\left(W_{\text {reg }}\right)(c)=-2 \pi \xi c_{1}(\mathcal{V})(c) .
$$

(3) Let us look now at the monotonicity for the gradient spheres. Let $\gamma$ be a gradient trajectory of $h$ between a fixed point $x_{0}$ of $V$ and a fixed point $q$ of $Q$. The circle action on $\gamma$ defines a gradient sphere $\mathbb{S}^{1} \cdot \gamma$. Its area is equal to

$$
[\omega]\left(\mathbb{S}^{1} \cdot \gamma\right)=2 \pi \int_{\gamma} i_{X_{h}} \omega
$$

where $X_{h}$ is the Hamiltonian vector field associated to $h$ (see for instance Audin [1, proof of Theorem VIII.1.1]). Moreover, as $\gamma$ is a trajectory of the gradient of $h$,

$$
\frac{d \gamma}{d t}=-\operatorname{grad} h=J X_{h}
$$

As a consequence,

$$
\int_{\gamma} i_{X_{h}} \omega=\int \omega\left(X_{h}, J X_{h}\right)=\int_{\gamma}\left\|X_{h}\right\|^{2}=\int\left\|\frac{d \gamma}{d t}\right\|^{2}
$$


is equal to the energy of this trajectory, that is,

$$
[\omega]\left(\mathbb{S}^{1} \cdot \gamma\right)=2 \pi\left(h\left(x_{0}\right)-h\left(\left(x_{q}\right)\right)=2 \pi(-\xi) .\right.
$$

Besides, the Chern class of this sphere is the difference of the sums of weights at $x_{0}$ and at $q$, that is,

$$
c_{1}\left(\mathbb{S}^{1} \cdot \gamma\right)=w(q)-w\left(x_{0}\right)=1
$$

(see Sections 4.1, A.2 and B.1), so that the gradient sphere satisfy

$$
[\omega](c)=-2 \pi \xi c_{1}(\mathcal{V})(c) .
$$

\section{References}

[1] M Audin, Torus actions on symplectic manifolds, revised edition, Progress in Math. 93, Birkhäuser Verlag, Basel (2004) MR2091310

[2] M Audin, On the topology of Lagrangian submanifolds. Examples and counterexamples, Port. Math. (N.S.) 62 (2005) 375-419 MR2191628

[3] M Audin, Lagrangian skeletons, periodic geodesic flows and symplectic cuttings, Manuscripta Math. 124 (2007) 533-550 MR2357797

[4] M Audin, F Lalonde, L Polterovich, Symplectic rigidity: Lagrangian submanifolds, from: "Holomorphic curves in symplectic geometry", (M Audin, J Lafontaine, editors), Progr. Math. 117, Birkhäuser, Basel (1994) 271-321 MR1274934

[5] P Biran, Quantum structures for Lagrangian submanifolds arXiv:0708.4221

[6] P Biran, Lagrangian non-intersections, Geom. Funct. Anal. 16 (2006) 279-326 MR2231465

[7] L Buhovski, Homology of Lagrangian submanifolds in cotangent bundles arXiv: math/0312265

[8] L Buhovski, The Maslov class of Lagrangian tori and quantum products in Floer cohomology arXiv:math/0608063

[9] J J Duistermaat, G J Heckman, On the variation in the cohomology of the symplectic form of the reduced phase space, Invent. Math. 69 (1982) 259-268 MR674406

[10] A Floer, Witten's complex and infinite-dimensional Morse theory, J. Differential Geom. 30 (1989) 207-221 MR1001276

[11] K Fukaya, Y-G Oh, H Ohta, K Ono, Lagrangian intersection Floer theory: anomaly and obstruction. Parts I-II, AMS/IP Studies in Adv. Math. 46, Amer. Math. Soc. (2009) MR2553465

[12] D B Fuks, V A Rokhlin, Beginner's course in topology: Geometric chapters, Universitext, Springer, Berlin (1984) MR759162 Translated from the Russian by A Iacob, Springer Ser. in Soviet Math. 
[13] F Lalonde, J-C Sikorav, Sous-variétés lagrangiennes et lagrangiennes exactes des fibrés cotangents, Comment. Math. Helv. 66 (1991) 18-33 MR1090163

[14] E Lerman, Symplectic cuts, Math. Res. Lett. 2 (1995) 247-258 MR1338784

[15] D McDuff, D Salamon, Introduction to symplectic topology, Oxford Math. Monogr., Oxford Science Publ., The Clarendon Press, Oxford Univ. Press, New York (1995) MR1373431

[16] J W Milnor, J D Stasheff, Characteristic classes, Annals of Math. Studies 76, Princeton Univ. Press (1974) MR0440554

[17] Y-G Oh, Floer cohomology of Lagrangian intersections and pseudo-holomorphic disks. I, Comm. Pure Appl. Math. 46 (1993) 949-993 MR1223659

[18] Y-G Oh, Addendum to: Floer cohomology of Lagrangian intersections and pseudoholomorphic disks. I [17], Comm. Pure Appl. Math. 48 (1995) 1299-1302 MR1367384

[19] Y-G Oh, Floer cohomology, spectral sequences, and the Maslov class of Lagrangian embeddings, Internat. Math. Res. Notices (1996) 305-346 MR1389956

[20] M A Ol'shanetskiü, A M Perelomov, A G Reĭman, M A Semenov-Tyan-Shanskiĭ, Integrable systems II, from: "Dynamical systems. VII: Integrable systems, nonholonomic dynamical systems", (V I Arnol'd, S P Novikov, editors), Encyclopaedia of Math. Sci. 16, Springer, Berlin (1994) 83-259 MR1256257

[21] P Seidel, Graded Lagrangian submanifolds, Bull. Soc. Math. France 128 (2000) 103149 MR1765826

[22] C Viterbo, Intersection de sous-variétés lagrangiennes, fonctionnelles d'action et indice des systèmes hamiltoniens, Bull. Soc. Math. France 115 (1987) 361-390 MR926533

Department of Pure Mathematics and Mathematical Statistics, University of Cambridge Wilberforce Road, Cambridge, CB3 OWB, UK

a.gadbled@dpmms. cam.ac.uk

http://www.dpmms.cam.ac.uk/ ag663/

Received: 5 March 2010 